\title{
THE KAPIIZA CONDUCTANCE OF THE (100) SURFACE OF COPPER AND POLYCRYSTALLINE INDIUM AND NIOBIUM
}

N. S. Snyder

Cryogenics Division

Institute for Basic Standards

National Bureau of Standards

Boulder, Colorado 80302

June 1975

Final Report Prepared for:

Office of Naval Research

Arlington, Virginia 22217 



\section{NBSIR $75-812$}

\section{THE KAPITZA CONDUCTANCE OF THE (100) SURFACE OF COPPER AND POLYCRYSTALLINE INDIUM AND NIOBIUM}

N. S. Snyder

Cryogenics Division

Institute for Basic Standards

National Bureau of Standards

Boulder, Colorado 80302

June 1975

Final Report Prepared for:

Office of Naval Research

Arlington, Virginia 22217

Covers April 15, 1971 to October 14, 1974

Contract Nos. NAonr-20-71, NAonr-8-72, NAonr-13-73, NAonr-22-74. Approved for public release; distribution unlimited.

Reproduction in whole or in part is permitted for any purpose of the United States Government.

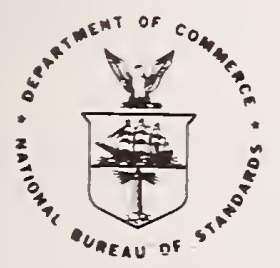

U.S. DEPARTMENT OF COMMERCE, Rogers C. B. Morton, Secretary 


\section{DISCLAIMER}

Certain commercial materials are identified in this report in order to adequately specify the experimental procedure. In no case does such identification imply recommendation or endorsement by the National Bureau of standards, nor does it imply that the material identified is necessarily the best available for the purpose. 
1. INTRODUCTION . . . . . . . . . . . . . . . . . . . . I

1.1. Background. The Acoustic Mismatch Theory. . . . . . . . . . 1

1.2. Previous Experimental Work. . . . . . . . . . . . . . . 2

1.3. Recent Theoretical Work. . . . . . . . . . . . . . 5

1.3.1. Modification of the Acoustic Mismatch Theory. . . . 5

1.3.2. Non-Continuum Approaches. . . . . . . . . . 7

1.4. Objectives of These Experiments . . . . . . . . . . . . . 8

2. DESCRIPTION OF APPARATUS . . . . . . . . . . . . . . . . . . 9 9

2.1. The Ultra-High Vacuum System. . . . . . . . . . . . . . 9

2.2. The Cryostat. . . . . . . . . . . . . . . . . 16

2.3. Electrical Measurements . . . . . . . . . . . . . . 17

3. EXPERIMENTAL PROCEDURES AND SOURCES OF ERROR . . . . . . . . . . . 17

3.1. Preliminary Sample Preparation. . . . . . . . . . . . . . 17

3.2. Sample Preparation in the UHV System. . . . . . . . . . . . 18

3.3. Cryogenic Procedures. . . . . . . . . . . . . . . . 19

3.3.1. "Storage" Vacuum. . . . . . . . . . . . . . 19

3.3.2. Thermometry . . . . . . . . . . . . . . 19

3.3.3. Parallel Heat Flow. . . . . . . . . . . 20

3.4. Analyses of Sample Properties . . . . . . . . . . . . . 22

3.4.1. Surface Area. . . . . . . . . . . . . . . 22

3.4.2. Analysis by X-Ray Techniques of Crystal Perfection. 22

3.4.3. Purity. . . . . . . . . . . . . . . 24

4. RESUltS OF MEASUREMENTS. . . . . . . . . . . . . . . . . . . 24

4.1. Corrections for Parallel Heat Flow. . . . . . . . . . . . 24

4.2. Copper Single Crystal Sample l. . . . . . . . . . . . . 25

4.3. Copper Single Crystal Sample 2. . . . . . . . . . . . 25

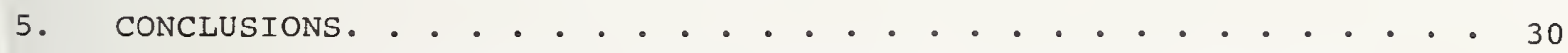

APPENDIX A. MEASUREMENTS OF THE CONDUCTANCE OF POLYCRYSTALLINE INDIUM • • • • • • • • • • • • • • • • • • • • • 32

APPENDIX B. MEASUREMENT OF THE CONDUCTANCE OF POLYCRYSTALLINE NIOBIUM • • • • • • • • • • . • • • • • . 36

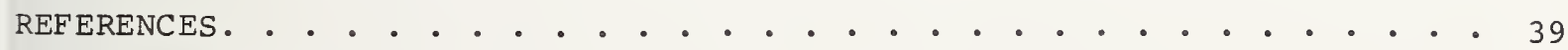

DISTRIBUTION LIST . . . . . . . . . . . . . . . . . . . 4 43 
Figure 1.

Figure 2 .

Figure 3.

Figure 4.

Figure $5 a$.

Figure $5 b$.

Figure 6.

Figure 7 .

Figure 8.

Figure 9.

Figure 10.

Figure 11.

Figure 12.

Figure 13.

Figure 14.

Experimental results and theoretical curves for

a copper-He II interface.

Schematic diagram of the vacuum system during

sample preparation.

Schematic diagram of the cryostat in which Kapitza

conductance measurements are made.

Photograph of apparatus.

Detailed view of sample chamber. . . . . . . . . . . . 14

Sample chamber in cryogenic mode.

Detailed schematic of the heat flow between the elements of the Kapitza conductance measurement system. . . .

Kapitza conductance measurements of single crystal

sample 1 .

Kapitza conductance measurements of single crystal

sample 2. • • • • • • • • • • • • . • • • • •

Comparison of measurements on annealed samples IC

and $2 \mathrm{~B}$.

Apparatus for Kapitza conductance measurements of

indium.

Kapitza conductance results for two polycrystalline

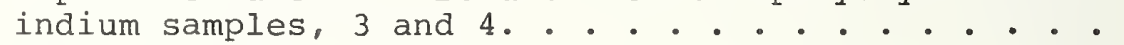

Comparison of Kapitza conductance measurements on indium with theory and with previous work.

Kapitza conductance of a polycrystalline niobium

sample.

Comparison of the observed Kapitza conductance of niobium with theory and with previous work. 


\section{ABSTRACT}

Measurements of the Kapitza conductance to liquid helium II across the (100) surface of single crystals of copper are presented. The temperature range of these measurements was 1.6 to $2.1 \mathrm{~K}$.

The sample surfaces were subjected to several different treatments. Some surfaces were cleaned by low energy argon ion bombardment, annealed in an ultrahigh vacuum system, and preserved under vacuum until purified liquid helium was admitted. Other surfaces were intentionally damaged by machining and/or exposure to the atmosphere. The conductance after these latter treatments was found to be about a factor of three higher than that of the more ideally cleaned and annealed surfaces, and a significant increase in the temperature dependence of the conductance was also observed. Indications are that these effects on the conductance are produced primarily by the last few atomic layers of the solid, so extensive cold-working, which impairs the thermal conductivity of the bulk, need not be undertaken to improve surface heat transfer. This finding has important implications for the design of practical heat transfer surfaces. Good reproducibility was found for the conductances of similarly treated sufaces and good correlation with studies of damage carried out by x-ray diffraction. The relationship of these results to the numerous current theories of the Kapitza conductance is discussed. Conductance measurements of polycrystalline niobium and indium are presented in the appendices.

Key words: Heat transfer to helium II; interfacial thermal resistance; Kapitza conductance; Kapitza conductance of copper; Kapitza conductance of indium; Kapitza conductance of niobium; (100) surface of copper; $x$-ray diffraction examination of single crystal lattice damage. 
THE KAPITZA CONDUCTANCE OF THE (100) SURFACE OF COPPER AND

POLYCRYSTALLINE INDIUM AND NIOBIUM

N. S. Snyder

\section{INTRODUCTION}

\subsection{Background. The Acoustic Mismatch Theory}

An analysis of the heat transfer between a solid body and liquid helium II shows that the thermal impedance may be separated into two parts. One thermal impedance is located precisely at the solid-liquid interface, and is described by the heat transfer coefficient, $h_{K}$, often called the Kapitza conductance. Thus, the heat flow, $\dot{Q}$, through the interface, is given by

$$
\dot{\mathrm{Q}}=\mathrm{h}_{\mathrm{K}} \mathrm{A} \Delta \mathrm{T} \text {, }
$$

where $A$ is the surface area of heat transfer, and $\Delta T$ is the temperature difference between the solid surface and the liquid. To restrict consideration to the interface proper and eliminate processes in the bulk liquid, one can think of $h_{K}$ as the limiting value reached as $\Delta T$ becomes very small, i.e.

$$
\mathrm{h}_{\mathrm{K}}=\lim _{\Delta \mathrm{T} \rightarrow 0} \frac{\dot{\mathrm{Q}}}{\mathrm{A} \Delta \mathrm{T}} .
$$

The second part of the thermal impedance occurs in the bulk liquid, helium II, and is negligible compared to the interfacial impedance under most conditions. In the temperature region near $1.9 \mathrm{~K}$, the Kapitza temperature drop across the interface is usually larger than the temperature drop across many meters of fluid, because of the very high thermal conductivity of helium II. For heat inputs of $1 \mathrm{~W} / \mathrm{cm}^{2}$, experimentally measured temperature differences across an interface range from about $0.3 \mathrm{~K}$ to $10 \mathrm{~K}$.

The Kapitza conductance is an even more important parameter at low temperatures because it varies with temperature approximately as $\mathrm{T}^{3}$, where $\mathrm{T}$ is the absolute temperature. Thus, the conductance is so large above liquid helium temperatures that the interfacial thermal barrier is usually not noticeable. On the other hand, at temperatures in the millikelvin region, the conductance may be so small that it is the limiting factor in the design of devices. But even at temperatures near the lambda point, the smallness of the conductance is quite noticeable, compared with the exceptionally high thermal conductivity of helium II. It was this factor which led to the original discovery of the interfacial thermal barrier by Kapitza. ${ }^{l}$ In fact, it was thought at first that the interfacial resistance was related to the superfluid properties of helium II, but conductances of similar magnitude were later found for solid- ${ }^{3}$ He interEaces, and these early theories were subsequently abandoned. ${ }^{2}$

Later, two possible reasons for the existence of interfacial thermal barriers at low temperatures were recognized. First, at temperatures far below the Debye temperature of a solid, only a relatively small number of phonons of high energy are available for heat transmission across an interface where electronic transmission is unimportant. (Because the magnitude of the Kapitza conductance is similar for metals and insulators, ${ }^{2}$ it is currently thought that electronic wave function "leakage" is relatively small, but see recent work of Wagner, et al. ${ }^{3}$ ) This effect, sometimes known as the phonon radiation limit, is demonstrated in a simple calculation that is found in reviews of the subject. 2,4 The conductance is 


$$
\mathrm{h}_{\mathrm{K}}^{\mathrm{PRL}}=\frac{2 \pi^{4} \mathrm{k}^{2}}{5 \theta_{\mathrm{D}}^{2} \mathrm{~h}}(3 \mathrm{~N} / 4 \pi)^{2 / 3} \mathrm{~T}^{3}
$$

where ${ }^{\theta} \mathrm{D}$ is the Debye temperature and $\mathrm{N}$ the number of atoms per unit volume. The $\mathrm{T}^{3}$ temperature dependence then results simply from the temperature dependence of the lattice portion of the specific heat at low temperatures, since this is a measure of the available energy.

A second reason for the interfacial thermal barrier is the large acoustic mismatch between liquid helium and most solids, which is expected to further reduce the phonon transmission. Since the distribution of momenta of phonons in the two media is so different, it is difficult to satisfy the laws of conservation of energy and momentum for arbitrary angles of incidence. This calculation was first carried out for liquid helium-solid interfaces by Khalatnikov, 5,6 and for solid-solid interfaces by Little. 7 The result in a form which may be compared with eq. (3) is

$$
h_{K}^{A M}=\frac{2 \pi^{4} k^{2}}{5 \theta_{D}{ }^{2} h}\left(\frac{3 N}{4 \pi}\right)^{2 / 3} \frac{4}{3}\left(\frac{3 \eta^{3}}{2 \eta^{3}+1}\right)^{2 / 3}\left(F_{1}+F_{2}\right) \frac{\rho_{1 i q^{c} l i q}}{\rho_{\text {sol } 1^{c} t}} T^{3},
$$

where $\eta$ is the ratio of the velocities of longitudinal and transverse waves, $p$ refers to the density, $c_{l i q}$ to the velocity of sound in the liquid, and $c_{t}$ to the transverse velocity of sound in the solid. $F_{1}$ is a measure of the heat flow due to phonon transmission within the allowed angle, and $\mathrm{F}_{2}$ is a measure of the heat flow due to the excitation of surface modes. (It is assumed that energy is effectively transferred from the surface modes to the bulk by some dissipative mechanism.) $F_{1}$ and $F_{2}$ are of approximately the same magnitude for most common solids, and the entire quantity between the square brackets is of order unity. Hence, a comparison of eq. (4) with (3) indicates that the main difference is in the appearance of the ratio of the acoustic impedance, $\rho c$, in the two media. This ratio is often about $10^{-3}$, which reduces the transmission significantly from the phonon radiation limit. However, for solid-solid interfaces, the mismatch is much smaller.

\subsection{Previous Experimental Work}

Quite a number of solids have been studied, usually in the polycrystalline state. Results are available for the more common structural metals, including aluminum, nickel, copper, and iron, as well as for the softer elements, such as tin, lead, and indium. Several dielectrics have been studied. The measurements up to about 1970 have been tabulated, ${ }^{2}$ and more recent results on aluminum and niobium, ${ }^{8}$ and iron, cobalt, and nickel ${ }^{9}$ are available. (The emphasis in this discussion will be on work in the temperature range near the lambda point; the present situation at lower temperatures is that relatively few experiments have been done, and the work has been carried out at a small number of laboratories, so there has not been much comparison of data by different workers.) The major conclusion of the higher temperature work has been that for both dielectrics and metals the observed conductances are far in excess of the Khalatnikov acoustic mismatch model, often by two orders of magnitude. 2,10 An example of the situation is shown in figure 1,11 which reproduces results for copper on polycrystalline samples. The results fall roughly in between the phonon radiation limit, eq. (3), and the acoustic mismatch theory, eq. 


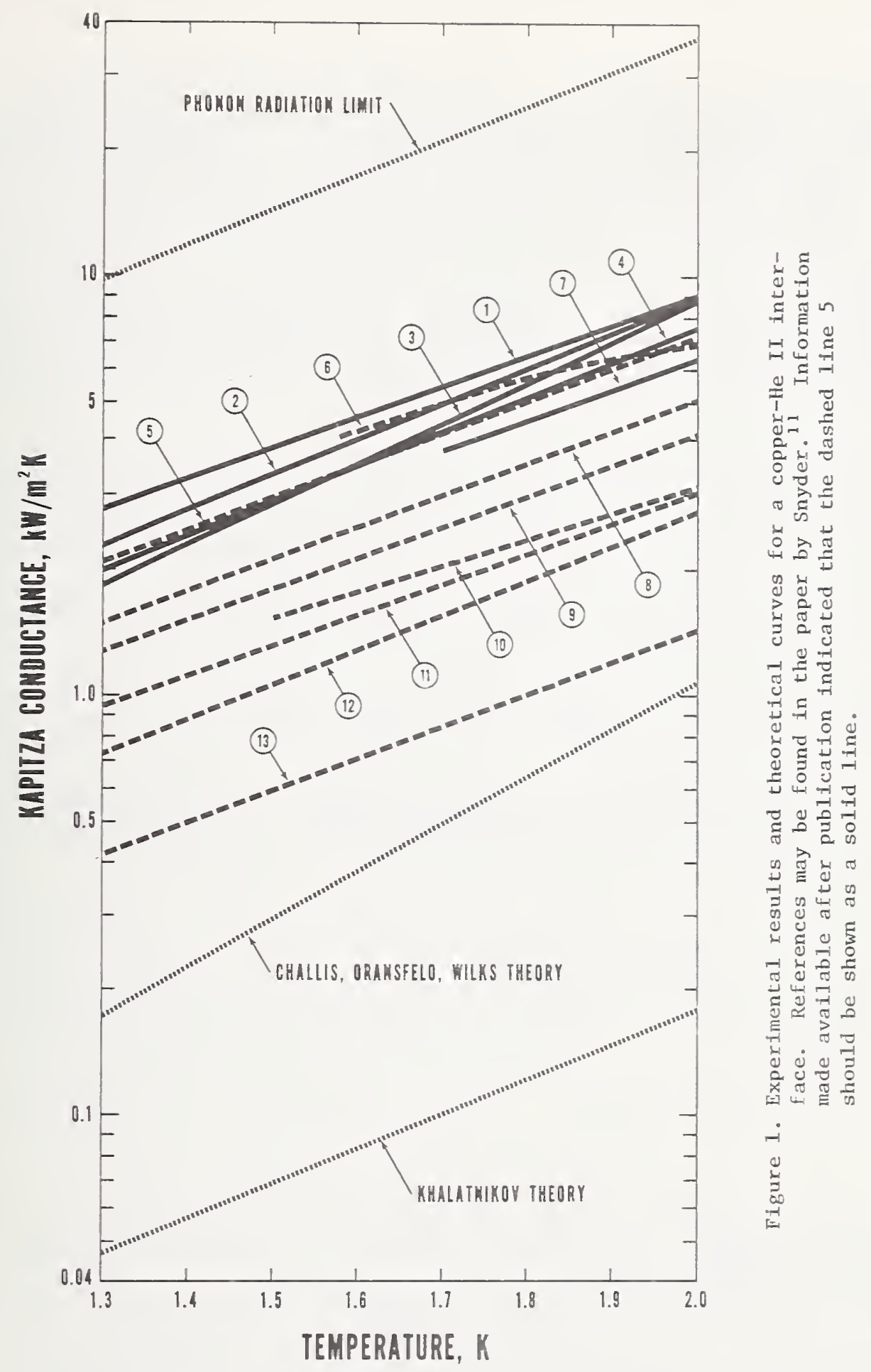


Furthermore, results by different workers are often in considerable disagreement. There seems to be a tendency for results on surfaces which were chemically etched before the measurements (solid lines) to exhibit a higher conductance than those which were simply machined (dashed lines). However, there are no results in which chemical etching or annealing were carried out on a sample which had been measured after it was machined, so it is difficult to assess the effect of cold work. Since the present work was started, some work along

these lines has been done at very low temperatures by Folinsbee and Anderson. 12 This will be discussed more fully below, but the applicability of these results to this temperature range is uncertain. The dependence on the Debye temperature and liquid density predicted by eq. (4) is also not observed experimentally.2

The characterization of the surfaces studied to date, in terms of the current knowledge of surfaces that has developed during the past decade, can only be described as extremely sketchy. Many of the early workers in the field (before about 1960, did not provide any description of surface treatment. More recently, typical metal samples have been machined from polycrystalline stock, mechanically polished, and sometimes etched or electropolished. Only a few samples have been annealed to remove damage. Exposure to the atmosphere has always occurred prior to the Kapitza conductance measurements of metals, except for work done by challis and cheeke ${ }^{13}$ in which ion bombarded samples of polycrystalline lead were kept in a vacuum of about $10^{-4}$ torr. Such a vacuum would not prevent an oxide layer from reforming on the surface. Also, polycrystalline copper samples were milled under liquid helium by Johnson and Little ${ }^{14}$ and then measured, but this procedure did not permit healing of the machining damage. Surface conditions have been, in general, better specified and more ideal for the few dielectrics, usually single crystals, that have been measured. There has been a measurement on a crystal of KCl cleaved under helium, ${ }^{15}$ and studies of silicon with differing amounts of surface damage have been carried out. ${ }^{14}$ The above studies, and others up to about 1970, are described more fully in table I of reference 2. More recently, cold-worked and/ or annealed polycrystalline copper exposed to the atmosphere has been studied 12,16 in the temperature range below $0.5 \mathrm{~K}$. Pumping on the sample surface for an extended period of time at room temperature has recently been found to affect the conductance subsequently measured at low temperatures. 17

It is unfortunate that the surfaces studied have not been better characterized, or more nearly ideal. The theory essentially is constructed for an ideal solid with a plane surface that is a "simple termination of the bulk", although a real interface has surface roughness and includes foreign atoms, dislocations, and other defects. Recently, some new theoretical effort related to non-ideal surfaces has been occurring, and this will be discussed in the next section. Before these modifications to the acoustic mismatch theory are considered, however, it should be mentioned that the theory apparently works for solid-solid interfaces at low temperatures, according to some work carried out in the past few years, chiefly by Peterson and Anderson 18 and by Cheeke, et al. 19 However, the applicability of the theory to solid-solid helium interfaces is still controversial, as data by Mezhov-Deglin obtained on a single crystal of ${ }^{4}$ He has a very different temperature dependence 20,21 than does data obtained by Folinsbee and Anderson. 22 Thus the data obtained by the first worker appear to be in reasonable agreement with theory at higher temperatures $(0.5$ to $2 \mathrm{~K})$, whereas the latter data do not.

It is appropriate to discuss several recent experimental studies of phonon transmission coefficients at this point, although a more detailed discussion will be deferred until the treatment of more recent theoretical results in the 
next section. These experiments involve efforts to measure, in principle, the transmission and reflection at an interface of phonons that are well-defined in terms of frequency, angle of incidence, and mode. Successive refinements in defining these parameters of transmitted phonons have recently occurred. of course in heat transmission, these parameters.are, in effect, averaged over frequency, mode, and angle by the phonon distribution for a particular solid at a given temperature. Guo and Maris ${ }^{2}$ measured the reflection of heat pulses from a dielectric crystal interface with liquid and gaseous helium and with solid neon, using a superconducting bolometer as a detector. The transmission found was much higher than predicted by the acoustic mismatch theory, and in fact was similar for solid neon and liquid helium, although the acoustic mismatch transmission factors are quite different. In experiments with helium gas adsorbed at the interface, transmission rose to the bulk liquid value when the film thickness was equal to about three layers of helium atoms. $A$ similar finding was reported by Long, et al. ${ }^{24}$ Kinder and Dietsche 25 used monochromatic phonon pulses of variable frequency, generated and detected by superconducting tunnel junctions. No structure at the maximum phonon and minimum roton frequencies is found in their results, though this would be expected on theoretical grounds if phonons were directly transmitted into excitations of the bulk liquid. 26 Thus, the microstructure of the first few layers of helium should be relevant to an explanation of the mechanism of the energy transfer at the interface, at least for temperatures above $\mathrm{l} \mathrm{K}$. This finding lends added interest to the present work, which is motivated by an attempt to characterize surface details on the atomic scale. The phonon transmission experiments, however, are apparently open to some uncertainty in that the transmission of energy directly into the liquid is not proven. 23 Also, there has not been much concern in the transmission work for layers of gases other than helium absorbed on the surface, although this may affect the results profoundly, because theoretical work at higher temperatures has shown that the properties of the adsorbate and its bond, rather than the bulk, control the energy and momentum exchange with incident gas particles. 27 These experiments have not been carried out at temperatures below the lamba point, where phonon energies are lower. An experiment by sherlock, et al. 28 which measured the angular distribution of the phonons radiated into the liquid from the (100) face of a sodium fluoride crystal indicated the presence of a sharp peak in the angular jistribution. This would tend to substantiate the acoustic mismatch theory because the requirement of conservation of momentum and energy leads to emission in a critical cone, but phonons reflected at larger angles were also observed, indicating a possible parallel mode of energy transmission.

\subsection{Recent Theoretical Work}

Much of the recent work in the field of Kapitza conductance has been directed towards modifications of the theory to account for the possible nonideal behavior of the actual interfaces used, rather than attempting the more difficult job of refining the experiments so that the surfaces employed would be more ideal and better defined. Hence, the available new theories suffer from the problem that direct quantitative comparison with experiment has been impossible, as will be seen in the detailed discussion below. A dichotomy of approach has occurred, with one school working on modifying the acoustic mismatch theory while still using the continuum approach (expected to be most applicable at temperatures less than $0.1 \mathrm{~K}$ ), and others attempting to discard the old theory entirely and to construct a new theory upon the basis of atomic interactions.

\subsubsection{Modification of the Acoustic Mismatch Theory}

The acoustic mismatch theory hypothesizes that heat transfer at the interface occurs only through phonons, and that most phonons incident on the interface will be reflected because of the large acoustic mismatch between liquid 
helium and most solids. But, if dissipative mechanisms such as scattering centers exist, the momenta and energy of some scattered phonons may be modified so that boundary conditions can be met, and the transmission will be

increased. 29 Khalatnikov actually took account of one type of dissipation, since his final result included the conversion of energy into the surface or Rayleigh modes, and it was assumed that all this energy was somehow absorbed into the bulk, 30 although the mechanism was not discussed. Early work on the interaction of conduction electrons with phonons by Andreev ${ }^{31}$ provided a possible mechanism. However, the Rayleigh wave mechanism increases the magnitude of the theoretical conductance only by a factor of about three. The theoretical curve shown in figure 1 includes this factor, which is not sufficient to bring about agreement with experiment.

In more recent work, phonons incident on the solid from the liquid outside the critical cone of acoustic-mismatch transmission are considered to create a non-propagating disturbance with an amplitude which decreases exponentially with distance from the surface much more rapidly than the Rayleigh waves do. When attenuation due to dislocations or other causes exists, energy can be absorbed from the surface disturbances into the bulk material. This mechanism has been examined by Khalatnikov and Adamenko, 32 Peterson and Anderson, 33 and Haug and Weiss. 34 The calculations employed perturbation theory and predicted that transmission would increase above the acoustic-mismatch model in proportion to the factor $\mathrm{p}=\lambda_{\mathrm{ph}} / l_{\mathrm{ph}}$ ' where $\lambda_{\mathrm{ph}}$ is the phonon wavelength, and $l_{\mathrm{ph}}$ is the phonon mean free path. There has been no independent way of determining ${ }^{2}$ '
so it has been used as an adjustable parameter to fit the theory to the " experiments. As Vuorio ${ }^{35}$ points out in his critique of these calculations, an assumption that $\mathrm{p}$ is temperature-independent, or nearly temperature independent, has to be made (though $l_{\mathrm{ph}}(\mathrm{T})$ varies with the type of lattice defect causing the scattering), and the probable variation of $p$ between samples is greater than the factor of 10-100 that is the range of the different experimental results for each metal. Challis ${ }^{36}$ felt that the smallness of ${ }^{p}$ ph required to explain the data was excessive, but this criticism has apparently been answered by Sluckin, et al. 37 and Peterson and Anderson ${ }^{33}$ who point out that the depth of penetration of the surface disturbances is relatively small (for example,

50 \& at $0.1 \mathrm{~K})$, so that the more familiar concepts of bulk attenuation are less relevant than surface attenuation, which could be very high. Experiments at low temperatures ${ }^{12}(<0.1 \mathrm{~K})$ showed qualitative evidence for the theory, although lack of reproducibility was a problem with the experiments. Whether the mechanism can explain the lack of agreement between the original acousticmismatch theory and experiment in the higher region of temperature that is of interest here is uncertain. Peterson and Anderson explicitly state that their calculations are expected to be valid only for $\mathrm{T}<0.1 \mathrm{~K}$, but Khalatnikov and Adamenko indicate their theory should also be valid up to the lambda point. Cheeke, et al. 9 failed to observe an increase in conductance in this higher temperature range after machining a sample that had been measured in the annealed state. In these experiments, mechanical polishing of the samples was followed by an anneal and a light electropolish. It is possible that defects and impurities from the mechanical polish could not be removed by the anneal, so that a sufficient number of defects were present in both cases. It is also possible that surface oxidation or physically adsorbed gases, which were present in both cases, were the determining factors in establishing the conductance. The data for lead seem to show the opposite effect, 11,38 and the previous results on copper seemed to also. 11 However, as noted above, the situation was unclear because comparisions had to be made between different samples measured by different workers. 
Another possible dissipative effect results from surface roughness. Little first pointed out that when the mean free path of the phonons is comparable to the interface roughness, the effective area will differ for phonons of different frequency: For long wavelength phonons the area is the plane area, but for short wavelength phonons the effective area will be the microscopic area. This can cause an increase in the exponent of temperature dependence, so that it is greater than three. Iittle 39 concluded that transmission would not be enhanced for the case of microscopic roughness of the size of the phonon wavelength. Adamenko and Fuks ${ }^{40}$ used different methods of approximation in their calculation of surface roughness, and concluded that when the phonon wavelength is comparable with the dimensions of the roughness, the scattering at the surface becomes nearly diffuse, so that surface waves can be transformed into volume waves, and transmission outsice the critical cone can be increased.

\subsubsection{Non-Continuum Approaches}

Because it appears that heat transfer across solid-solid interfaces does follow an acoustic mismatch relationship, a theoretical approach which exploits some unique property of ${ }^{3} \mathrm{He}$ and ${ }^{4} \mathrm{He}$, perhaps in both the liquid and solid state, might be fruitful. The acoustic theory assumes that both materials are continuous media with properties which change discontinuously at the interface to their bulk values. However, neglect of Van der Waals forces in a relatively open system such as liquid helium may be unjustified. A number of calculations which attempt to take such factors into account have appeared recently. There was also an early attempt by Challis, et al. 41 to consider the improvement in acoustic matching due to the increased helium density near the interface, which results from consideration of Van der wals forces between the helium atoms and the solid. The effect was not large enough to account for the experimental results (see fig. l). More recent efforts included dynamic effects of the Van der wals forces as well as static effects in a calculation patterned after early calculations of the gas accommodation coefficient. 42 Again, however, the effect found was not large enough to bring about agreement with experiment because the contribution to the conductance does not start to be important at

a low enough temperature. Vuorio 43 considered that improved acoustic matching in the right temperature range might occur if the interfacial layer contained defects or impurity structures such as oxides that had vibrational modes of frequencies different from those of an ideal interface, perhaps as low as $100 \mathrm{GHz}$. The simplified model for the calculation consists of a layer of solid atoms separated from the bulk solid by a layer of liquid atoms. An enhanced transmission of about a factor of 10 above the acoustic mismatch model is found. This factor of 10 would not be large enough to explain many of the experimental results, but this could be the result of using a simplified model. Transmission from the liquid to the solid does not occur outside the critical cone, which avoids some theoretical paradoxes. Long 44 and weiss ${ }^{45}$ have recently undertaken calculations of energy transfer by desorption of helium atoms and obtained some qualitative agreement with the coefficient of reflection found from the transmission experiments. The weak temperature dependence may be explained by such calculations.

Opsal and Pollack 46 have blended the two approaches discussed above -attenuation in the solid at low temperatures and impedance matching at higher temperatures due to the increased density of helium near the surface. Their theory has an adjustable parameter for each of these situations, and can be made to fit some of the previously published data. However, there have been no measurements on a single sample over the entire temperature range from the millikelvin region to the lambda point, and there is considerable variation now being found in the magnitude of results at millikelvin temperatures, 47 so whether this approach has general applicability is unclear. For example, as 
mentioned earlier, studies in the transition region from high to low temperatures, $20,22,48$ have given results for the temperature dependence that varied from approximately $\mathrm{T}^{4}$ to $\mathrm{T}^{2}$.

Saslow $^{49}$ has raised the question of dissipation in the liquid as a possible source of increased energy transmission. He has pointed out that. the dominant frequency of the thermal phonons, even at helium temperatures, is too high for the application of the usual two-fluid model of the hydrodynamics of liquid helium. (In the two-fluid theory, the helium below the lambda point is considered to be a mixture of two components: normal fluid, which carries entropy and has a normal viscosity; and superfluid, which carries no entropy and has zero viscosity. The collision time, $\tau$, between excitations in the twofluid model is too long for this type of liquid to respond to the thermal vibrations of the solid, so this model is not applicable to the Kapitza conductance problem.) An intermediate region would exist for frequencies slightly larger than $1 / \tau$, in which the behavior of the fluid may be described as viscoelastic in an analogy with ordinary fluids such as glycerine. As the frequency is raised, however, an atomic description becomes necessary. Saslow assumes that at the thermal frequencies of helium II, a viscoelastic atomic description is appropriate, and carries out calculations on this model which blend features of both the viscoelastic and atomic regime. He finds that viscous dissipation is an important source of energy available for transfer from the liquid to the solid, but does not obtain an exact solution for the proportion of this energy which is transmitted and contributes to the Kapitza conductance. To do this would require a solution of the Boltzmann equation for atoms rather than phonons, which is a difficult theoretical problem. Because of the short distances over which energy is dissipated, the dynamics of atomic collisions probably determine the energy transfer. This approach should give similar results for ${ }^{4} \mathrm{He}$ and ${ }^{3} \mathrm{He}$, in accordance with the experimental results that conductances to both liquids are similar.

\subsection{Objectives of These Experiments}

The above discussion illustrates the variety of present theoretical approaches. The fact that Kapitza conductance experiments, especially on metals, have not really provided a test of the Khalatnikov theory or other theories because the surfaces were not sufficiently well-defined has been the motivating factor for the experiments described here. The objective of these studies was to do experiments under controlled conditions in an effort to indicate whether acoustic mismatch or an atomic interaction approach is most applicable. Attempts to improve the Kapitza conductances of practical devices operating below the lambda point are likely to be tedious and unproductive until more basic knowledge of the problem is available. The difficulty with past experiments has been that too many possible variables were poorly defined, and where attempts were made to vary parameters, more than one was varied simultaneously, so results were inconclusive. The experiments described here constitute a step forward towards the goal of measuring the Kapitza conductance of an atomically clean, structurally undamaged surface. The techniques used for sample preparation were those that have been developed in surface studies using low energy electron diffraction (LEED), although the present apparatus does not incorporate such devices for in situ surface characterization. A single crystal sample was prepared in an ultrahigh vacuum (UHV) environment by using low energy argon ion bombardment to clean the surface and high temperature annealing to reduce damage. The sample was then sealed against an indium o-ring. A high vacuum was maintained at the sample surface while the apparatus was converted to a cryostat for the measurements of the Kapitza conductance to helium II. Measurements of the Kapitza conductance were made over the temperature range of 1.6 to $2.1 \mathrm{~K}$.

LEED and Auger spectroscopy studies have shown, over approximately the last decade, that many metals, insulators, and ionic crystals have a clean surface structure that is essentially a "simple termination of the bulk", in which the lattice spacing for the final few layers of atoms is larger than the bulk spacing by only a few percent. However, the surface atoms may have a much larger 
amplitude of vibration than the bulk atoms do. 50 In contrast, semiconductors with smaller coordination numbers for surface atoms and a more open lattice exhibit rearrangements of the surface that have fewer "dangling bonds" than the idealized structure. 51 such clean surfaces are frequently prepared in a UHV environment with the pressure below $5 \times 10^{-10}$ torr, using cycles of low energy argon ion bombardment followed by annealing to remove embedded argon atoms and superficial damage resulting from bombardment. The annealing is desirable because ion bombardment does not necessarily leave a smooth surface. It is usually necessary to use a low index face of a single crystal to get an ideal surface. Although contamination of the order of $10 \%$ of a monolayer of foreign atoms is difficult to detect with LEED, the identity of surface contaminants can be determined with Auger spectroscopy, in which the energy of electrons from atoms in the surface is detected. It was not feasible to use either of these two techniques in this experiment, since to do so would mean a considerable increase in the complexity and expense of the apparatus. It seemed fruitful, however, to do an experiment in which the procedures for obtaining clean surfaces that were established by LEED and Auger studies would be used, and the sample maintained in a UHV environment until purified liquid helium was admitted to make the cryogenic measurements. This procedure should result in the first Kapitza measurements on a metal surface that has a low dislocation density, a fairly planar surface (so that the microscopic area is close to the macroscopic area), and little contamination (perhaps several monolayers at most) of foreign atoms. Since a monolayer can be deposited on a fresh surface in only one second in a vacuum of $10^{-6}$ torr, preservation of such a well-prepared surface in a much better vacuum is essential if heavy contamination is to be avoided. A shiny metal surface prepared by a chemical etch and immediately installed in a cryostat will still have an oxide layer some tens of angstroms deep, as well as possible impurities from the etch and some physisorbed atoms, in spite of its bright appearance. Also, to study the effect of damage in the layers next to the surface, it is desirable to have the capability to remove this damage by first bombarding the surface to remove foreign atoms, so that subsequent annealing does not simply diffuse impurities into the surface where they may interfere with recovery. Because of the strong probability that atomic collision calculations will be necessary to resolve the theoretical problems, it seems highly desirable at this point to make further observations of Kapitza conductance only on well-defined single crystal surfaces.

In brief, the present studies with single crystal samples of copper showed that operations which tended to make the surface more ideal decreased the conductance toward the original Khalatnikov acoustic-mismatch theory. Introducing damage increased the conductance substantially, as did an oxide layer. An attempt was made to vary only one parameter at a time in the course of the measurements. The state of the last few atomic layers of the solid seemed to have the predominant influence on the size of the conductance.

\section{DESCRIPTION OF APPARATUS \\ 2.1. The Ultrahigh Vacuum System}

The ultrahigh vacuum system is constructed of welded stainless steel, with commercial flanges which are sealed by copper gaskets. All metal valves with metal bellows and soft metal seats are used. A well-trapped diffusion pump is supplemented by a titanium sublimation chamber. The system is bakeable. Equipment for ion bombarding and annealing the sample is included. Figure 2 gives a schematic diagram of the system, while the size and relationship of the cryostat and other components are shown in the photograph, figure 4 . A commercial titanium sublimation cartridge, $A$, is mounted in a chamber, $B$, in which the walls are cooled by liquid nitrogen. This increases the efficiency of hydrogen pumping 52 which is important because hydrogen is expected to be a major constituent of the gas load. The insulating vacuum space outside the wall enclosing the 


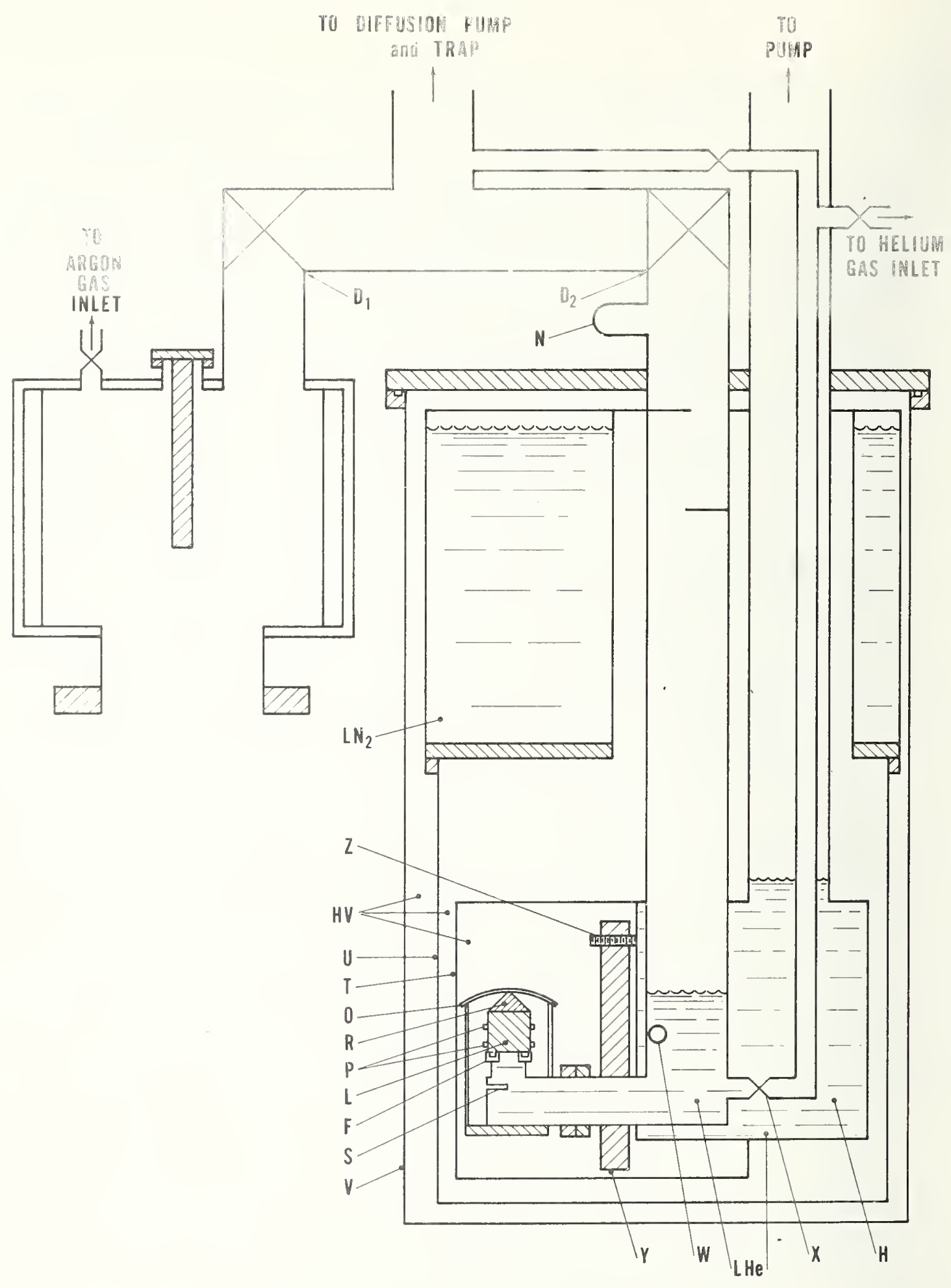

Figure 2. Schematic diagram of the vacuum system during sample preparation. $A$, titanium sublimation cartridge; B, sublimation chamber; $C$, sample chamber; $D_{1}, D_{2}, 50 \mathrm{~mm}$ gold-sealed valves; $E$, tubing to which sample is sealed after preparation; F, indium 0-ring groove; G, continuation of tubing to which sample is sealed after preparation; $H$, pumped helium bath; J, Ion gun; $K$, sample manipulator; $I$, sample; $M$, radiation shield; $\mathrm{N}$, vacuum gauge; $\mathrm{S}$, well; $\mathrm{W}$, level indicator of tantalum wire; $\mathrm{X}$, . hellum Inlet valve; $Y$, sample chamber flange. 


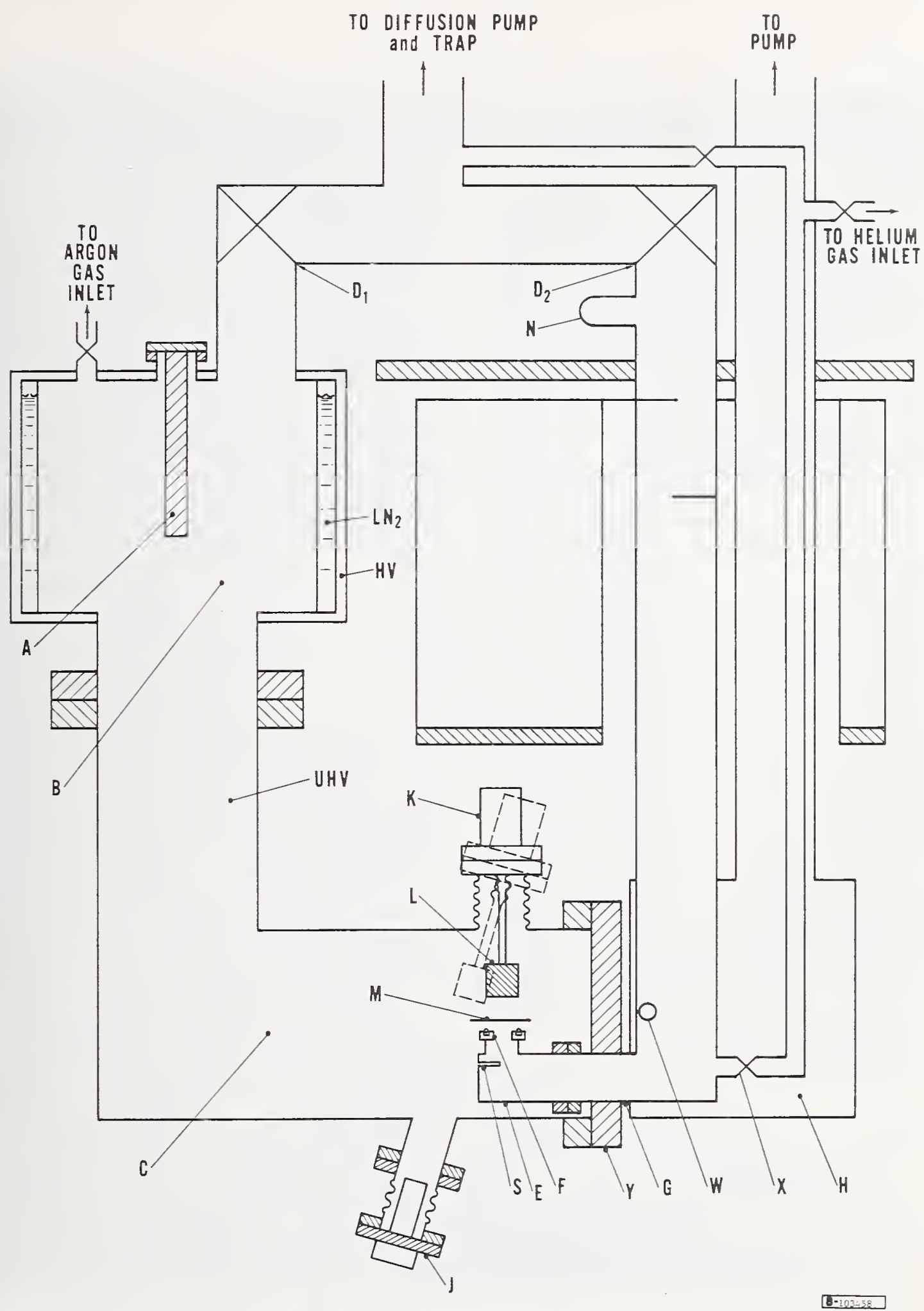

Figure 3. Schematic diagram of the cryostat in which Kapitza conductance measurements are made. $D_{1}$ and $D_{2}, 50 \mathrm{~mm}$ gold-sealed valves; $F$, indium O-ring groove; H, pumped helium bath; L, sample; $N$, vacuum gauge; 0 , harness which seals sample; $P$, split rings to which thermometers are attached; $R$, cone on which heater is wound; $S$, well for germanium resistance thermometer; $\mathrm{T}$, radiation shield at liquid helium temperature; $U$, radiation shield at liquid nitrogen temperature; $V$, outer wall of cryostat; $W$, level indicator (tantalum wire); $X$, helium inlet valve; $Y$, sample chamber. 


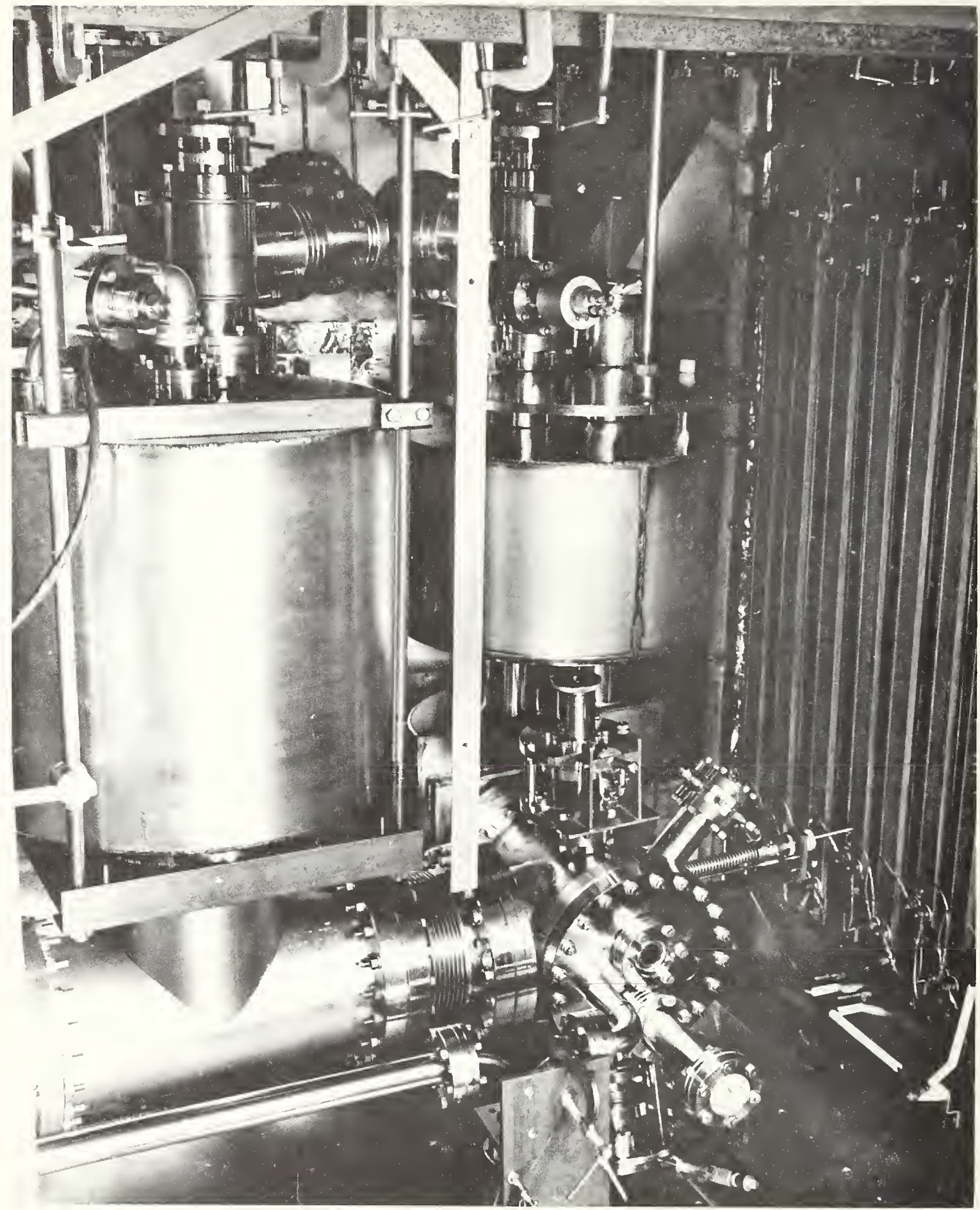

Figure 4. Photograph of apparatus. The sample chamber is at the lower right, the cryostat is at the upper right, the titanium sublimation pump is on the left, and valves and connections to the diffusion pump are at the top. 
nitrogen is separate from the UHV system. The sublimation chamber is connected to the sample chamber, $C$, by an elbow which is $150 \mathrm{~mm}$ in diameter. A baffle (not shown) is a further precaution against titanium entering the sample chamber. The sublimation pump is backed by a $100 \mathrm{~mm}$ oil diffusion pump. A cold cap helps reduce backstreaming, and a commercial high conductance trap with a constant cold zone at liquid nitrogen temperature prevents migration of any oil molecules into the system. The trap was kept filled with liquid nitrogen by an automatic system for the duration of the experiments. The forepump is equipped with a metal mesh trap. The entire vacuum system, except for the trap and pump, is mounted in an oven with removable walls, so that the system can be baked to $400^{\circ} \mathrm{C}$. The sublimation pump chamber is baked by operating the cartridge at high power. The argon and helium gas lines leading to the system can be evacuated and heated. The argon gas is further purified as it passes through the sublimation chamber. Two high conductance gold seal valves, $\mathrm{D}_{1}$ and $\mathrm{D}_{2}$ ' allow the sample chamber to be pumped via two paths: by the higher speed titanium sublimation pump during sample processing, or by the diffusion pump alone. One nude ionization gauge (not shown) measures pressure in the sample chamber. Another ionization gauge is indicated by $\mathrm{N}$.

The sample chamber contains an inner tube, $E$, with an elbow on which is located a groove, $F$, for an indium o-ring. Tubing $\mathrm{E}$ is connected by a commercial flange to tubing $G$ which passes through the cryostat helium bath, $H$, and on to valve $\mathrm{D}_{2}$. The apparatus is designed to accept cylindrical samples $25 \mathrm{~mm}$ in diameter and 19 to $38 \mathrm{~mm}$ in length. The outer bellows on the sample manipulator, $K$, is used to position the sample, $L$, for ion bombardment. The dashed outline in figure 2 indicates the position for ion bombardment. An ion gun, denoted by $J$, is mounted on a bellows that allows it to be moved through an angle of a few degrees to cover the entire surface of the sample. A second, inside bellows on the sample manipulator allows the sample to be raised and lowered. Electrical contact to the radiation heater which is used to anneal the sample is made through a feedthrough that is also located on the sample manipulator. The heater is not shown in the schematic figure 2 , but is shown in more detail in figure $5 \mathrm{a}$. It consists of tungsten wire which passes through ceramic spacers which surround the upper two-thirds of the sample. The heater assembly is enclosed within a molybdenum shield. The heater is capable of raising the sample temperature to about $700^{\circ} \mathrm{C}$ in about an hour. A thermocouple placed above the sample gives an approximate measure of the temperature to within about $25^{\circ} \mathrm{C}$, but avoids contamination of the sample from surface diffusion at high temperatures.

Details of the sample sealing arrangement are shown in figure 5 , since the seal involved some development work. It was considered undesirable to machine the sample because of the damage that would be introduced, although this would have simplified the design of a reliable seal. Care is needed to line up the sample manipulator with the o-ring groove. The flange which mates with flange $Y$ is rotatable, as is the smaller flange welded to tubing $E$ (fig. 2). The sample chamber is aligned with a substitute sample when it is bolted to the cryostat before each measurement. A beryllium copper contact ring with many "fingers" that form an inverted cone helps to position the sample as it is lowered to the o-ring. The beryllium copper contact ring is located on a split ring support so that it can be removed after the sample is sealed. However, as the sealing force is applied, some rotation is allowed for at the bearing-nut junction. Also, there is flexibility in the sample support because it has been made partly of thin walled stainless tubing to allow enough thermal isolation of the sample for annealing. Thus, during the application of sealing force, the sample surface can align itself parallel to the surface in which the o-ring groove is cut. Some preliminary experiments on seals showed that this provision was essential for a plane sealing surface. A spring outside the vacuum system provides the sealing force of about $300 \mathrm{~N}$. An indium o-ring is used to take up the sealing force and minimize strain damage to the sample. The sealing force is about $20 \mathrm{MPa}$ if it is assumed that all force is concentrated on the inner edge of the groove, which is $0.25 \mathrm{~mm}$ wide, and $3 \mathrm{MPa}$ if the force is distributed over the entire groove 


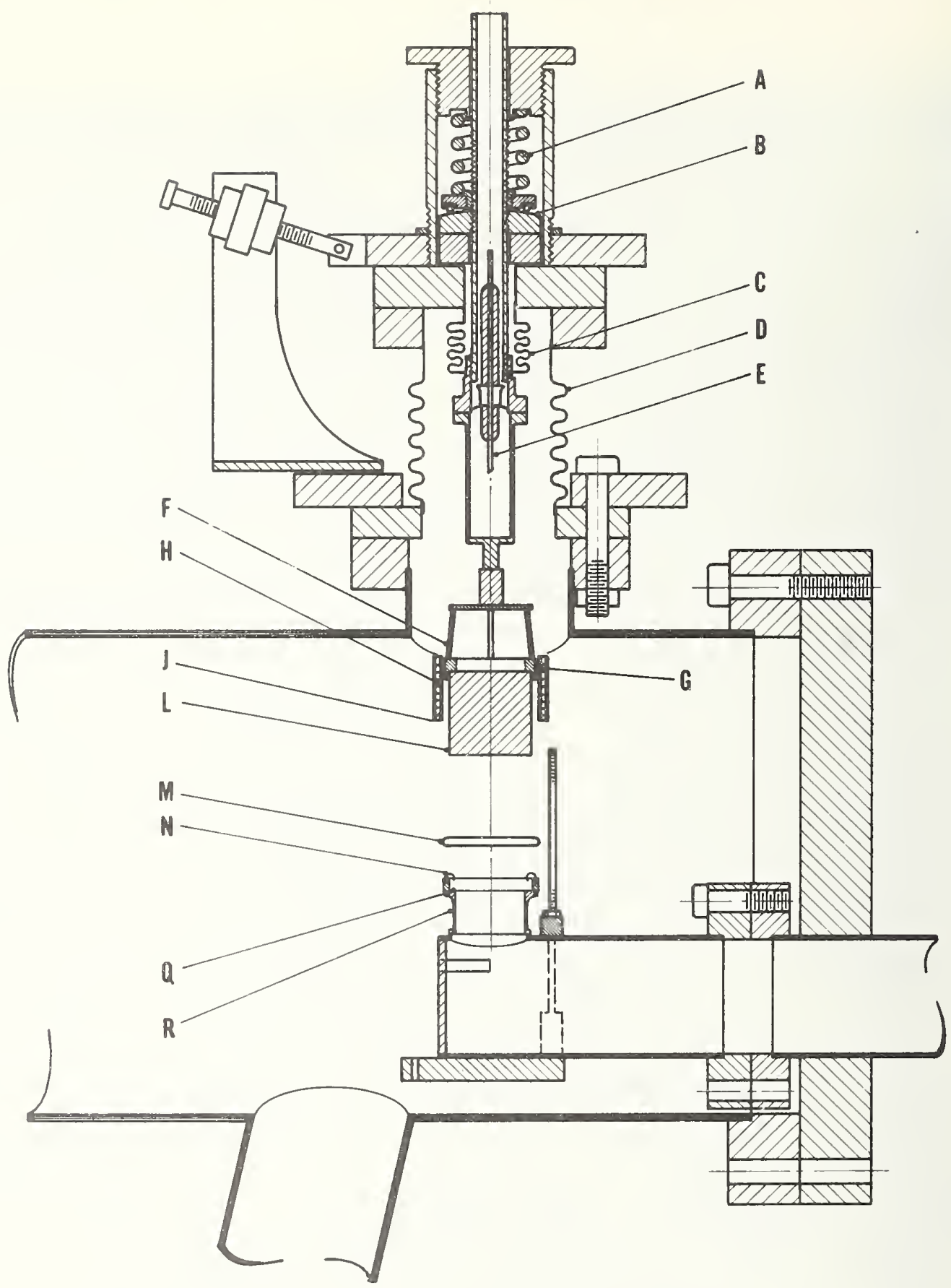

Figure 5a. Detailed view of sample chamber. A, beryllium copper spring; B, nut which applied sealing force; C, inner bellows; D, outer bellows; E, electrical feed through (connection to heater not shown); F, supports of thin walled tubing; G, sample holder; H, sample heater; J, molybdenum radiation shield for heater; $L$, sample; $M$, radiation shield; $N$, contact ring guide; $Q$, indium O-ring groove; $R$, thin-walled stain- . less steel support. 


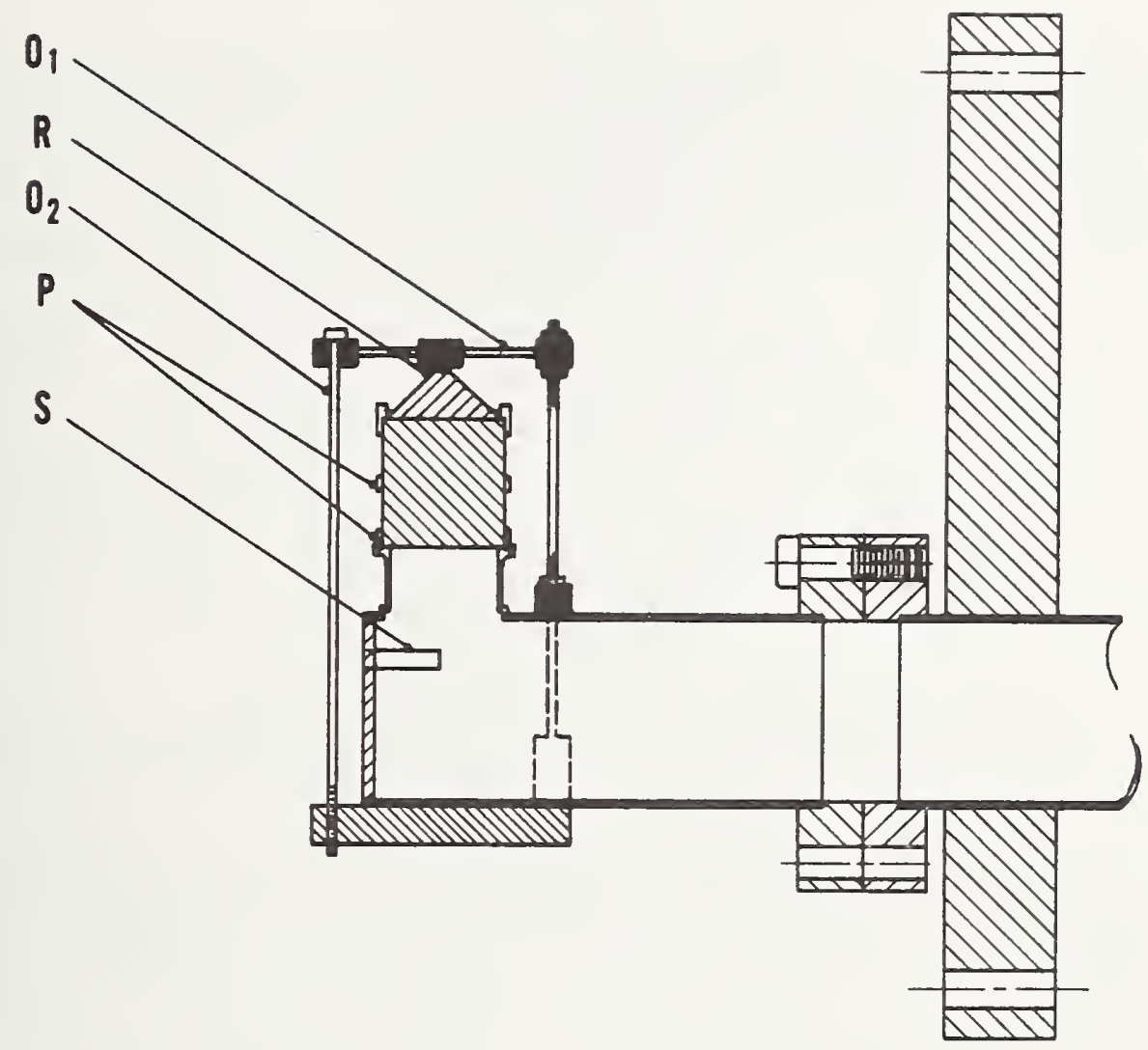

Figure 5b. Sample chamber in cryogen1c mode. $O_{1}$, beryllium copper rods; $R$, cone on which heater is wound; $\mathrm{O}_{2}$, harness arrangement which seals the sample; P, split rings of copper to which the carbon resistance thermometers are attached; $S$, wel1 in which germanium resistance thermometer is inserted. 
area. Observations have been that the indium adheres quite strongly to a position on the sample which corresponds to the inner edge, so the higher figure may be more correct. Samples were examined by x-ray diffraction (Sec. 3.4.2) after sealing, to check for damage.

\subsection{The cryostat}

Most of the cryostat is constructed of welded stainless steel, except for the radiation shields which are copper. All welds were checked individually on a sensitive leak detector during the course of construction and rewelded if necessary. Repeated thermal shocking to liquid nitrogen temperatures was part of the testing procedure. Consequently, there were no problems encountered with superleaks in the welded or gasketed joints despite the large number of thermal cycles from about $500 \mathrm{~K}$ to $2 \mathrm{~K}$ during the course of the measurements. Part of the cryostat remains in place during the bakeout of the UHV system and the processing of the sample, as may be seen by comparing figure 3 with figure 2. After the sample is sealed, valve $\mathrm{D}_{1}$ is closed, but the sample surface is still pumped upon by the path leading through valve $\mathrm{D}_{2}$. Air is admitted to the system and the sample chamber access flange (not shown) is removed. The sealing force is then transferred from the sample manipulator to a harness arrangement, 0 , indicated schematically in figure 3 and in detail in figure $5 \mathrm{~b}$. Two beryllium copper rods $\left(O_{1}\right.$ in $\mathrm{fig}$. $\left.5 \mathrm{~b}\right)$ now provide the spring force as $\mathrm{O}_{2}$ on the harness is tightened slightly. The three supporting pillars connected to the sample manipulator are positioned so that the cone, $R$, which transmits the force to the sample, and the beryllium copper rods can fit between them. These supports are then detached from the sample holder and raised by the sample manipulator. The sample chamber is next unbolted from the flange $Y$ and withdrawn.

The guide for the sample sealing operation is then removed, and the thermometers, which are mounted on split copper rings, $\mathrm{P}$, are attached to the sample. Carbon resistors are used for thermometers on the sample. One lead of each thermometer is soldered to the clamp to improve thermal contact. The heater is located on the cone, $R$, which is already in place. A germanium resistance thermometer is installed in the well, S, of the sample chamber by using vacuum grease for thermal contact. (This, of course, has to be thoroughly cleaned before the next UHV operation.) A germanium thermometer is used because it is desirable to know the absolute temperature of the helium bath at the sample surface, without the complication of opening this clean system to an oil manometer, since oil migration in a cold trap is difficult to avoid. A liquid helium level detector, $W$, consists of a $70 \mu \mathrm{m}$ diameter tantalum wire which becomes superconducting in the liquid but not in the gas if sufficient current is supplied to it. This detector, which is wound upon a glass cylinder, is bakeable and compatable with the UHV system. It remains in place throughout the experiment. Because the large $20 \mathrm{~cm}$ flange $\mathrm{Y}$ that remains attached to the helium bath did not cool down as well as expected (it is welded to tubing of small diameter), copper screws coated with indium on the ends are threaded through the bolt holes to touch the bath wall, as shown by $z$. Radiation baffles for the sample helium bath are already in place in the neck of the sample chamber as shown in figure 3. They are designed not to reduce the pumping speed appreciably.

After the sensors are in place, the rest of the cryostat is assembled. The helium temperature radiation shield, T, is bolted to the cryostat bath, H. A coat of indium on the contacting surface of the shield of annealed copper improves thermal contact. The thermometer leads are tempered to this shield with a polyimide varnish that has been baked on and withstands thermal cycling well. A nitrogen-temperature shield, U, also made of copper, is bolted to the bottom plate of the nitrogen bath. Again, lead wires have been tempered to this shield. The outer chamber of the cryostat, $V$, can be raised by a pulley arrangement and bolted on to the top flange of the cryostat. A smaller flange at the bottom contains feedthroughs for the electrical leads. Both plates are sealed by rubber o-rings. The cryostat is pumped out by a separate diffusion pumping 
system to a vacuum in the $10^{-7}$ torr range. A sensor resistance thermometer and a heater for temperature control are placed in the bath, $H$. A helium level detector mounted on the end of a thin tube is also placed in this bath. The cryostat is precooled by admitting liquid nitrogen to space $\mathrm{H}$ and waiting several hours for the large mass of shields, cryostat walls, and flanges to cool. Then the cryostat is pressurized to blow out the nitrogen, helium is transferred to chamber $H$, and helium gas from a storage cylinder is admitted to the sample bath via tubing that passes first through a liquid nitrogen trap and then through coiled tubing in bath $\mathrm{H}$. The helium input lines are heated during each bakeout to remove impurities. Further impurities should be removed in the tubulation at helium temperature. (Coiling is not shown in the schematic diagram for simplicity.) Valve $\mathrm{X}$ is provided in the event it is necessary to warm up the system again to repair some cryogenic problem. Then most trapped impurities will be prevented from migrating to the sample chamber.

The pumped chamber of the cryostat holds about seven liters of liquid. with a loss rate of about 0.5 liters/hr., there are about eight hours for data accumulation after a helium transfer and pumpdown. Since it was necessary to make wall thicknesses relatively large to prevent permeation into the vacuum system and to support the heavy vacuum flanges, this loss rate was expected. The sample chamber helium bath is about $0.05 \mathrm{~K}$ warmer than the pumped bath, and this was expected. Design here was obviously a compromise because the vertical tubing to the bath had to be made large enough to allow a good pumping speed at the sample after sealing, yet a large diameter here allows more superfluid helium film flow and more radiation and conduction heat leaks.

\subsection{Electrical Measurements}

An electronic temperature controller stabilizes the temperature of the pumped bath, which in turn stabilizes the sample bath. At each temperature, zero power readings are obtained from the two carbon thermometers and the germanium thermometer. Then the change in thermometer resistance is measured for several power inputs to the heater. The germanium thermometer was calibrated separately and rechecked during the course of the measurements. There is a three wire connection to each carbon thermometer, with the current being returned through the common ground. Current is supplied to each thermometer in turn, only as it is read. Power levels to the thermometers were less than a nanowatt. Measurements are made on a digital voltmeter with a dc system in which the current is reversed to cancel extraneous emfs. Heating power measurements were obtained by measuring the voltage across the heater, using a separate set of voltage leads, and the current into the heater resistance.

\section{EXPERIMENTAL PROCEDURES AND SOURCES OF ERROR}

\subsection{Preliminary Sample Preparation}

The procedure used in cutting a sample to size is important if excessive damage is to be avoided. Conventional methods of cutting can introduce dislocations up to a depth of several mm in copper, ${ }^{53}$ and even spark cutting results in some damage. 54 Sample 1 was cut by the supplier, semi-elements, Inc., by an abrasive string saw and etching procedure. Sample 2 was spark cut, according to the supplier, Aremco Products, Inc. The heat transfer face of sample 2 was rough cut in this laboratory by spark cutting, followed by heavy etching and electropolishing. About $0.5 \mathrm{~mm}$ was removed. The specified orientation of the heat transfer face was (100) to within $2^{\circ}$ for both samples. While this was not checked precisely, analysis of the perfection of the crystal, with the $\mathrm{x}$-ray diffractometer (described below) indicated that there could not have been any significant deviation from this orientation. The purity of sample 1 was specified as $99.995 \%$, and sample 2 was specified as $99.999 \%$ pure. A rough measure of the thermal conductivity of the samples was obtained as a by-product of the Kapitza conductance measurement, and this indicated that the conductivity of sample 2 was higher by about a factor of two.

Further smoothing of the sample surfaces was accomplished by electropolishing, except for measurement 1A. This surface was prepared by an automatic 
mechanical polisher using diamond abrasives of $30 \mu \mathrm{m}$ and $15 \mu \mathrm{m}$ size. Diamond abrasives are thought to cut away high regions readily and thus cause less damage than soft abrasives. Final polishing was done with $0.3 \mu \mathrm{m}$ alumina abrasive. This mechanical polishing was followed by chemical polishing intended to remove some of the damage and imbedded impurities from the polishing. The solution contained 22\% $\mathrm{HNO}_{3}, 54 \% \mathrm{H}_{3} \mathrm{PO}_{4}$, and $24 \%$ acetic acid, and was used at a temperature between 60 and $70^{\circ} \mathrm{C}$. The total amount removed was $40 \mu \mathrm{m}$. Siome pitting occurred with a pit size of several microns. This was seen only in the single crystal sample and not in the polycrystalline sample holder, and so may have been due to impurity inclusions in the sample.

The other surfaces investigated, $1 \mathrm{~B}$ through $1 \mathrm{~F}$, and $2 \mathrm{~A}$ to $2 \mathrm{C}$, were prepared to a mirror finish by electropolishing. 55 The amounts removed were 0.1 to $0.2 \mathrm{~mm}$ each time. The electrolyte consisted of a $60 \%$ solution of phosphoric acid with cuo added at the concentration of about $15 \mathrm{~kg} / \mathrm{m}^{3}$. The cupric ions suppress the liberation of hydrogen gas at the cathode, so that the cathodic overvoltage does not change with time, and a stable polishing condition is obtained. A cell voltage of about $1.10 \mathrm{~V}$ was set by measurement at the sample surface with a copper reference electrode. The sample is placed in a jig which serves as a guard to prevent rounding of the edges. The bottom of the beaker which is used for the electropolishing was ground flat, because the flatness of the surface obtained on the sample is similar to the uniformity of the electropolishing cell. After the electropolish, a rinsing solution of $5 \% \mathrm{H}_{3} \mathrm{PO}_{4}$ is used, followed by deionized water. A final, very brief (about 20 seconds) chemical polish was carried out just before the sample was installed in the apparatus. This was followed by rinses in deionized water, and in highly filtered methyl ethyl ketone, after which the sample was blown dry in a stream of nitrogen gas.

\subsection{Sample Preparation in the UHV System}

The sample chamber and its components, including the indium o-ring, are cleaned carefully before each run. The usual cleaning procedure consists of vapor degreasing in hot trichloroethylene, agitation in a detergent solution in an ultrasonic bath, numerous rinses with hot water followed by deionized water, and finally, a rinse with highly filtered methyl ethyl ketone followed by drying in a stream of nitrogen gas. The last procedure was necessary because the apparatus was not located in a clean room, although a clean bench could be used for preliminary assembly work. All work in the sample chamber was carried out with degreased tools and nylon gloves.

After assembly, the system is evacuated to a pressure in the $10^{-8}$ torr range by the diffusion pump, and baked out for about 24 hours. The maximum temperature in the oven was about $190^{\circ} \mathrm{C}$, but the temperature of the indium, which is located near the bottom of the oven, remains below the melting point of $156^{\circ} \mathrm{C}$ because there is no forced air circulation in the oven. Bakeout is followed by outgassing of the filaments and heater, and bakeout of the titanium sublimation pump. After operation of the sublimation pump, the pressure in the sample chamber falls to the $10^{-10}$ torr range as measured by the nude ionization gauge mounted in the sample chamber. The upper glass gauge reads a slightly higher pressure. (Gauge pressure was not corrected for the residual gas composition, which was not determined. This would not change the pressure by more than a factor of 2.) After a good vacuum is achieved, research grade argon (99.9995\% purity) is admitted, and ion bombardment is started in the tilted position. Typical ion bombardment parameters were: $300 \mathrm{~V}, 8 \mu \mathrm{A}$, pressure $2 \times 10^{-4}$ torr, for a time of 20 minutes, followed by an anneal at $650^{\circ} \mathrm{C}$ for 1 to 2 hours. This procedure is usually carried out twice. Specific information for each sample is shown later in the table of section 4. According to published figures for yields, 56 an argon ion bombardment current of $8 \mathrm{\mu A} / \mathrm{cm}^{2}$ for $1 / 2$ hour should remove a thickness of approximately $0.6 \mu \mathrm{m}$ from a smooth, electropolished surface. During the anneal, the sample 
is returned to the vertical position so that the radiation shield for the indium will be effective. The purpose of the anneal is to remove argon gas that may have diffused into the sample, and to heal the moderate damage to the surface layer that may have occurred during the low energy bombardment. As noted earlier, a thermocouple was placed just above the sample to minimize contamination. Visual examination of the glowing sample gave an approximate verification of the readings. Annealing temperatures for each measurement are noted in the table.

\subsection{Cryogenic Procedures \\ 3.3.1. "Storage" Vacuum}

After the final anneal, some hours must elapse before the sample is cool enough to seal because thermal conductances from the sample to other parts of the chamber are sma 11. During this time, the vacuum is in the low $10^{-10}$ torr range. This is the range that, from LEED studies, is usually found to be adequate for preservation of a clean surface for a period of hours. The minimum monolayer time at $10^{-10}$ torr is 3.6 hours, if all incident molecules stick to the surface. However, after the sample was sealed, the vacuum at the heat transfer surface was not as good as initial tests had indicated. This reading, indicated in the table in section 4 , was usually in the $10^{-8}$ or upper $10^{-9}$ torr range. There is some possibility that this reading may have been more a characteristic of the gauge tubulation than of the pressure seen by the sample, since the pressure, measured at room temperature, did not decrease as the cryostat was cooled to liquid helium temperatures. The gauge is connected by $18 \mathrm{~mm}$ I.D. tubing to the $50 \mathrm{~mm}$ tubing that connects the sample to the pumping system. After the seal is made, 6 to 8 hours are normally spent in transferring the sealing force, removing the sample chamber, attaching thermometers, and assembling and pumping out the cryostat, during which the sample is exposed to a vacuum in the $10^{-9}$ torr range. Iiquid nitrogen is then transferred into the pumped helium chamber. After overnight cooling at liquid nitrogen temperatures, followed by several hours of helium gas cooling of the rather massive $(20 \mathrm{~kg})$ system, liquid helium was transferred into the pumped chamber, and then the gaseous helium was condensed into the sample chamber through the system of traps described earlier. Thus the sample surface was in a low temperature environment for 20 to 24 hours before measurements were actually underway. In some cases, cryogenic problems arose during the cooldown and an additional storage time occurred. This is indicated in the table. It is not known with certainty whether the vacuum was better than $10^{-9}$ torr during this period. It is felt that this cooling probably reduced the pressure at the sample surface, though, as noted above, this was not reflected at the room temperature gauge. Molecules from the rest of the system should be trapped out to some extent before reaching the sample surface, and local outgassing from the walls surrounding the sample should be reduced. These effects are counterbalanced by observations that surfaces held at low temperatures generally seem to deteriorate faster, 57 presumably because of the higher sticking probability at low temperatures. On the other hand, Rhodin 8 has observed that oxidation of copper surfaces is retarded at low temperatures, and Lee and Farnsworth ${ }^{59}$ felt that true oxidation of a cleaned surface did not occur except in the presence of sizeable amounts of impurities, such as water molecules.

\subsubsection{Thermometry}

Before the cryostat was given a final cleaning and attached to the UHV system, several measurements were made to verify that the cryostat would perform as desired to make Kapitza conductance measurements. For these tests, the sample bath was connected to a sensitive oil manometer that could measure temperatures below the lambda point to within approximately $0.001 \mathrm{~K}$. It was found that the germanium resistance thermometer, mounted in a well extending 
into the sample chamber as described in section 2.2, followed the sample bath temperature quite closely as heat was put into the sample heater, or as the pumped bath temperature was changed. The temperature measured with the germanium thermometer is offset from the sample bath temperature by an amount which varies slightly from run to run, probably depending upon the thermal contact achieved with the vacuum grease. The offset varies with bath temperature during a given run, from about $0.035 \mathrm{~K}$ at $2.1 \mathrm{~K}$ to about $0.045 \mathrm{~K}$ at $1.5 \mathrm{~K}$. This change is not expected to seriously affect the in situ calibration of the carbon resistance thermometers at zero power, since the slope $\mathrm{dR} / \mathrm{dT}$ of the calibration curve does not change significantly over $0.010 \mathrm{~K}$, and it is this quantity which is involved in determining the differences in temperature that occur when heat is applied. In two of the measurements the leads to the thermometer developed faults at low temperatures, and so the bath vapor pressure was measured using a temporary connection to an oil manometer with a liquid nitrogen trap. No significant differences were found in the temperatures determined this way, as compared to those determined with the germanium thermometer.

Another question about the thermometry is whether an annular ring on the outside of the sample can accurately measure the temperature distribution on a plane through the crystal when the length-to-diameter ratio is close to one. Because of the smallness of the phonon mean free paths and high conductivity, this is thought to be the case for the present system, for in principle, trouble would only be encountered for certain alloys with a small electronic thermal conductivity, yet high mean free path for phonon collisions with electrons. 60 Also, a check was made by measuring the second sample, 2, of twice the length-to-diameter ratio of ID-IF. The distance of the sensors from the heater was also increased on this sample. Good reproducibility was found, as discussed later in section 4 .

The thermometer voltages and currents were read on a digital voltmeter that has a quoted instrumental accuracy of $\pm 0.3 \mu \mathrm{V}$. Noise and variations in time of the thermal emfs resulted in a total estimated inaccuracy of about 1.5 $\mu \mathrm{V}$ in each primary voltage measurement. After a number of operations on these primary numbers, the resulting error in the measured temperature difference at the interface ranged from about 1 to $15 \%$, depending on the conductance of a given sample and the power level applied to it.

\subsubsection{Parallel Heat Flow}

Figure 6 is a schematic diagram of the various heat flow paths discussed below. The heat flow from the heater through the supports which furnish the sealing force was measured and found to be negligible: It was less than $0.5 \%$ of the heat flow through the sample surface. The heat loss through the thick part of the stainless steel tubing in which the indium groove is cut amounts to about 2 \% of that passing through the sample area exposed to helium. This correction is calculated using the recent measurements of cheeke, et al. ${ }^{9}$ as an estimate for the conductance, and using fin calculations. 61 Calculation shows heat leaks along the lead wires of $0.08 \mathrm{~mm}$ manganin to be negligible. Since these leads were coated with a thin superconducting layer of lead-tin solder, Joule heating would also be expected to be negligible.

The greatest problem with parallel heat leaks occurred because the indium from the seal tended to extrude along the sample to a much greater extent than had been anticipated. Separate measurements, without UHV sample preparation, were made with a soldered joint instead of an indium joint for a comparison, and these confirmed the importance of the effect. The Kapitza conductance of two samples of the polycrystalline indium used in the seal (99.99\% purity, from the Indium Corporation of America) was therefore measured in separate experiments. These experiments indicated that the correction from the indium could be as much as $20 \%$. Details of these measurements are given in appendix A. In making the correction to the data for the heat flow through the indium, uncertainties arise from two factors: the difficulty in measuring the area of the extruded indium exactly, and the uncertainty in the value of the Kapitza 


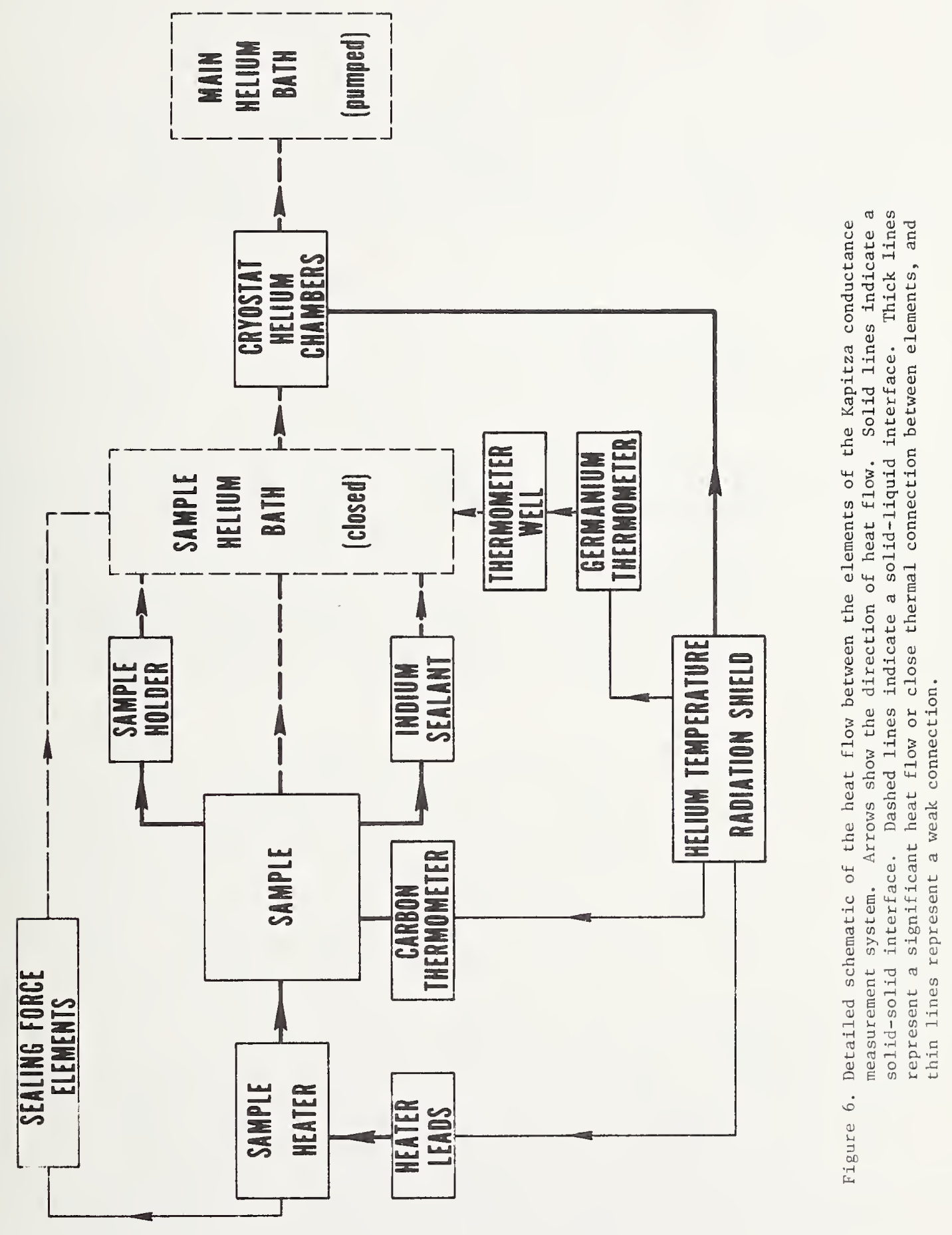


conductance of the indium. The latter uncertainty is by far the most important. The area of the indium was determined by a photographic technique and was found to be nearly the same in all cases. The expected uncertainty due to the indium conductance is determined from the results on the two samples measured, and is included in the error bars shown on the plots of the results. However, since the indium used is from the same roll of wire (except for run $2 \mathrm{~A}$ where a different roll was inadvertently substituted) and undergoes the same. treatment in each measurement, results for different measurements can be compared in a relative sense. One aim of the experiments was to see whether better agreement could be found with the ideal acoustic-mismatch theory by better surface preparation, and since previous experiments were in disagreement by an order of magnitude or more with this theory, an absolute uncertainty of 10 to $20 \%$ was not of great concern. The effects of different surface preparation were far larger than this.

\subsection{Analyses of Sample Properties}

\subsubsection{Surface Area}

The area of the exposed copper in the sample was taken to be the geometrical area. Absorption studies have indicated that for similar methods of preparation of metal surfaces, the enhancement over the geometrical area is not large. 57,58 This factor would not, therefore, be sufficient to reduce the conductance more than 10 to $20 \%$, which would not result in any significant change in agreement with theory. Sample 1 had more surface structure, of the order of micrometers, than did sample 2 after polishing. Pits and small hillocks, of about 10 to $50 \mathrm{\mu m}$ size, developed at scattered points on the surface, covering perhaps 2 to 4 \% of the total area. These structures did not appear on the polycrystalline sample holders that were used in the polishing processes to prevent rounding of the edges, and they were also almost entirely absent on sample 2, so it is believed that they might have been nucleated by impurities or defects in sample 1. Sample 2 appeared to be without structure when examined under a metallurgical microscope; that is, on a scale of the order of $5000 \AA$.

\subsubsection{Analysis by $x$-Ray Techniques of Crystal Perfection}

After the first few measurements, the sample was examined by Laue back reflection photography. Although the spots were sharp, indicating a good surface layer, the results were only qualitative, and numerical comparisions were difficult. After some of the later measurements, it was possible to examine the sample on an x-ray diffractometer. The radiation used was the cobalt $K \alpha$ line, which has a penetration depth in copper of about $14 \mu \mathrm{m}$. Significant differences in the linewidths of the (200) reflection were found, which seemed to be well-correlated with surface treatment. The results apply only to a spot about $3 \mathrm{~mm}$ in diameter, approximately in the middle of the heat transfer surface. After measurement $1 C$, on a very well-annealed sample, the width at half height, in terms of $\theta$ (the angle of reflection) was $0.032^{\circ}$ for the electropolished and ion bombarded surface, and $0.060^{\circ}$ for the opposite surface, which was untreated and subject to pressure from the heater cone. It was not possible to directly examine the spark-cut surface of sample $2 \mathrm{~A}$ due to scheduling problems, but the matching surface of the small piece that was cut away from this sample had a width of $0.12^{\circ}$. The actual surface $2 \mathrm{~A}$ was probably in somewhat better shape than this because it was heavily electropolished. However, these values give some indication of the improvement of the surface lc over a spark-cut surface. The sample was examined after run $2 \mathrm{~B}$ and found to have a width of $0.035^{\circ}$, which shows an interesting agreement with IC, since these two surfaces were prepared as identically as possible. Finally, the intentionally damaged sample ID was examined and found to have a width of $0.12^{\circ}$. These results are included in the table. It should be noted that these linewidths have a relative significance, but not an absolute meaning, because there was no absolute calibration in terms of beam width, etc. Also, of course, the sampling depth may be much deeper than is relevant for the actual mechanism of energy transfer, since it is about 300 times larger than 


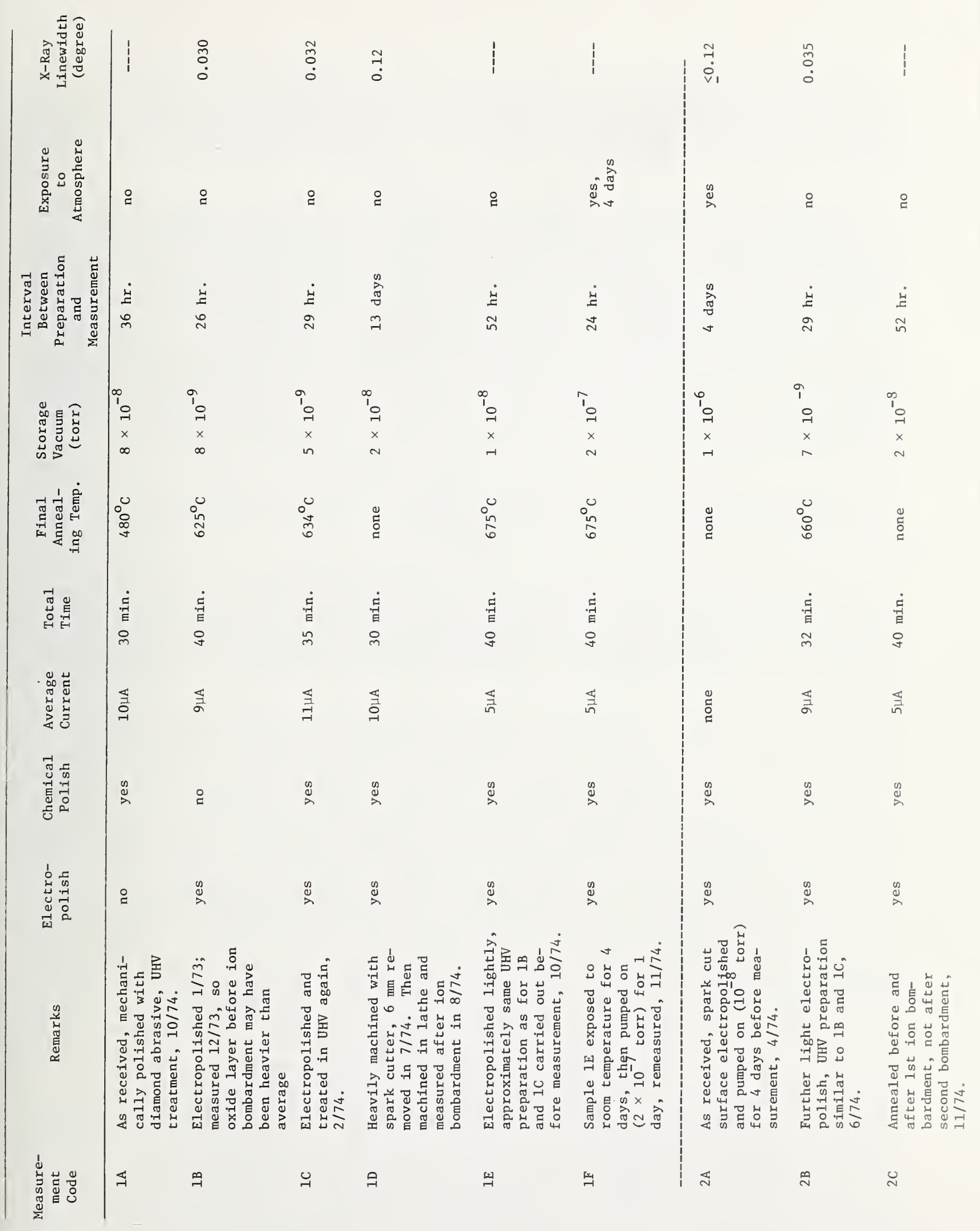


the phonon wavelength in the copper at $2 \mathrm{~K}$, and as indicated earlier, the relevant depth may be considerably less than even this. Nevertheless, the results of the analysis are mentioned because of the good correlation with the measurements, as discussed below.

\subsubsection{Purity}

The eddy current decay method ${ }^{62}$ was used to obtain the ratio of the specimen resistance at $273 \mathrm{~K}$ to its resistance at $4 \mathrm{~K}$ (RRR). This nondestructive method is particularly useful for large, relatively pure samples. The high temperature resistivity is characteristic of the base metal, but the low temperature resistivity reflects the impurities and physical imperfections of the individual sample. Both samples had been annealed before examination to remove as many physical defects as possible. of course, this method is sensitive to only impurities which act as electron scatterers, and thus impurities such as iron and the transition metals affect the RRR most strongly. The stated purity of sample 1 was 99.995\%, according to the supplier, and its RRR was 70. The RRR of sample 2 was 380 , which is significantly higher, and its stated purity was $99.999 \%$. As will be seen in the next section, this difference in the RRR did not appear to affect the Kapitza conductance.

\section{RESUITS AND MEASUREMENTS}

Before examining the results in detail, the procedure for going from the raw data to the information presented in the figures will be discussed. Several corrections to the data must be calculated.

\subsection{Corrections for Parallel Heat Flow}

The heat transfer through the indium is subtracted from the power input, using an average value for the indium conductance based on the results given in appendix $A$. The much smaller parallel flow through the stainless support tubing is also subtracted. For each temperature, a plot of the interfacial temperature difference against power per unit area of copper is prepared. (This quantity is corrected for the $3 / 2(\Delta T / T)$ effect when this factor is appreciable. ${ }^{2}$ ) It is the slope determined from these points that is plotted in the figures as $h_{K}$. No effect of high power analogous to that reported by Cheeke, et al. ${ }^{63}$ was seen in the $\Delta T$ plots, but the inaccuracy in $\Delta T$ may have been large enough to obscure this. Also, power densities in this work ranged from 5 to $40 \mathrm{~mW} / \mathrm{cm}^{2}$, whereas the anomaly was observed by comparing the slope below about $5 \mathrm{~mW} / \mathrm{cm}^{2}$ with the slope at higher power levels. If undetected in this data, the anomaly could result in an underestimation of $h_{K}$ by about $25 \%$. In the present studies, only equilibrium measurements were made, in contrast to the system employed by Cheeke, et al. in which power slowly increased with time as a ramp function. For some very high power inputs, equilibrium could not be obtained because of bath temperature drift, and so these data were discarded. A high power effect for densities of about $40 \mathrm{~mW} / \mathrm{cm}^{2}$ was observed in the measurements on indium, where the geometry was more like that of Cheeke's group, but it was eliminated by simple precautions (see appendix A).

The errors shown in the figures are due to the inaccuracies in temperature measurement, plus the uncertainty in the indium conductance as estimated from the two samples measured. Each effect contributed about half of the error. of course the indium uncertainty, unlike the temperature jump uncertainty, is not random, and would have to lie in the same direction for each temperature point of a given set of measurements. In evaluating the random scatter of the data for a given sample, then, the errors should be mentally djvided in half. To allow for the uncertainty of the indium conductance, however, the whole curve should be shifted up or down by about half of the error. Another error is not shown in the figures because it is roughly similar for almost all points. This is the uncertainty of about $0.02 \mathrm{~K}$ in the temperature readings of one set of measurements relative to another, due to the somewhat indirect method of deriving the bath temperature. However, temperatures for 
samples IC and ID were obtained from the vapor pressure of the sample bath, and so should be more accurate. For these measurements, the system was opened to the manometer only after the liquid helium had been condensed into the sample chamber. Since the slopes of IC and ID are quite different, lack of accuracy in temperature measurement clearly is not responsible for this effect.

\subsection{Copper Single Crystal Sample 1}

Figure 7 shows the data obtained with sample 1, after various types of surface preparation. The abcissa of this and the following figures is the average temperature of the bath ana solid. The data separate into two groups of high and low conductances, which also exhibit high and low exponents of temperature dependence, except for sample lE. The difference in conductances between the two groups is a factor of 2 or 3 . In the higher grouping, measurement IA was obtained after the sample had been mechanically polished, as gently as possible, and then chemically polished and ion bombarded and annealed in vacuum. However, the annealing temperature after the final ion bombardment was $480^{\circ} \mathrm{C}$, which was not as high as the temperatures of over $600^{\circ} \mathrm{C}$ for the other anneals. Measurements $1 \mathrm{~B}$ and $1 \mathrm{C}$, which showed a much lower conductance, were made more than a year later, so some healing may have occurred at room temperature in that time interval. A similar phenomenon may have been seen by Anderson and Johnson 16 at low temperatures. Measurement ID, which closely reproduces curve lA, was made after severe mechanical damage had taken place. An ion bombardment was carried out to remove surface impurities, but the sample was not annealed after this. The sample surface had been exposed to air in situ for several days at room temperature before measurement $1 F$ was made. It also lies in the upper group. However, except for this exposure, conditions were identical to measurement $1 \mathrm{E}$, on an annealed surface, which preceded $\mathrm{IF}$, and which lies in the lower group. The fact that nothing was changed between these two measurements, except that the sample surface was exposed to the air, tends to eliminate various spurious causes of the pronounced differences in magnitude between the two groups, such as unintentional variations in sample sealing forces. The sample surface was pumped on at room temperature for approximately a 24-hour period before measurement $I F$ to remove the more loosely adhering molecules from the surface.

Annealing preceded all the measurements in the lower group of conductances. Final anneal temperatures ranged from about 625 to $675^{\circ} \mathrm{C}$. Also, measurements $\mathrm{IB}$ and $\mathrm{IC}$ were made after the sample had been heavily electropolished $(0.025 \mathrm{~cm}$ removed each time), so it would be expected that most mechanical damage introduced into the surface by the mechanical polishing had finally been removed. The effects of damage and annealing are seen to be approximately reversible: The lowest conductance obtained for an annealed sample, measurement 1C, was followed by the highest, ID, after the sample had been heavily damaged. Then, after measurement ID, further electropolishing and annealing returned the sample to a conductance that was lower by a factor of two, although it does not have the small slope of IC. This may have occurred because all the dislocations introduced could not be removed. It should also be noted that before sample ID was machined heavily, about $6 \mathrm{~mm}$ were inadvertantly spark planed off the sample, yet subsequent measurements approximately reproduced the curve for lA. This, and the subsequent reversibility shown by lE, tends to diminish the possibility that a different impurity composition of the sample in its interior could account for the differences in conductance, because the results do not seem to be a function of depth.

\subsection{Copper Single Crystal Sample 2}

Figure 8 supports similar conclusions for sample 2, though the measurements on this sample were less extensive. Measurement $2 \mathrm{~A}$ was made on a spark cut surface, though much of the damage was undoubtedly removed by the heavy electropolishing that was necessary to reduce the somewhat rough cut to a smooth surface. Also, this sample had a layer of oxide, plus whatever impurities remained after the chemical polish and final wash, although it was placed under vacuum within about an hour after this procedure. An oxide layer of about

$20 \AA$ would be expected to form ${ }^{64}$ during this time. Thus, this sample is similar 


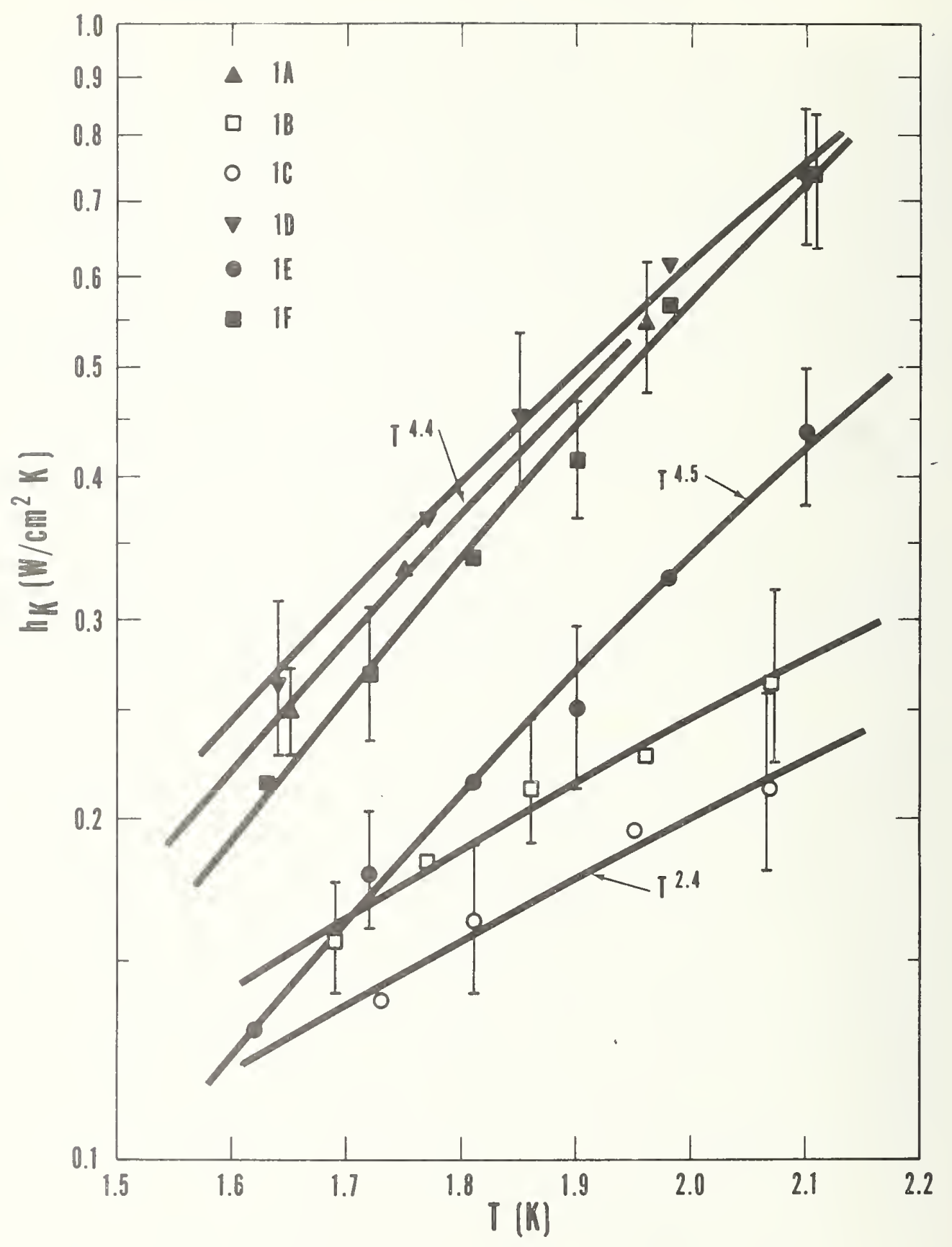

$75 \times 4266$

Figure 7. Kapitza conductance measurements of single crystal sample 1. Symbols are identified in the text and the table. 


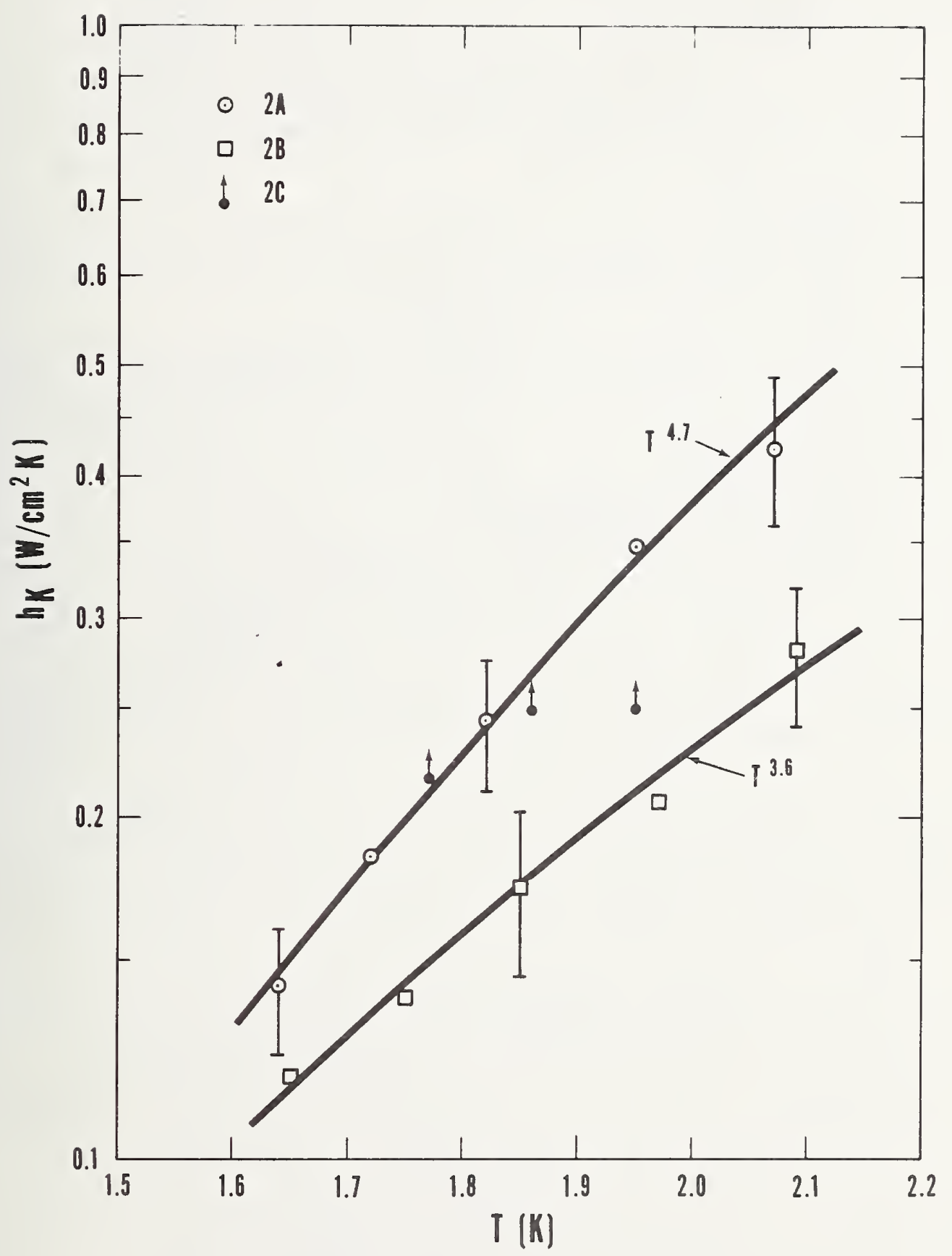

$[\overline{75 \times 4765}$

Figure 8. Kapitza conductance measurements of single crystal sample 2. Symbols are identified in the text and the table. 
to $1 \mathrm{~F}$ except for probable damage. The sample received further electropolishing and the usual UHV treatment before measurement $2 \mathrm{~B}$. The conductance was lowered by a factor of two at $1.9 \mathrm{~K}$, and again the exponent of temperature dependence also decreased. These results are similar to those for sample 1 . Good agreement is found between measurements $1 C$ and $2 B$, as would be expected, since the surface preparation conditions for these two measurements were as identical as possible. The two measurements are compared in figure 9, which shows the good reproducibility. The small lack of agreement in the slopes may indicate only the uncertainty in measuring that quantity in the present setup, due to both the use of a thermometer well and to the lack of a suitable number of points over a wide temperature range.

The table shows that the x-ray linewidths, where available, correlate with the observed conductances. This is true in spite of the fact that the linewidth is composed partly of contributions from layers far below the surface layer that would not be expected to affect the conductance.

It had not been foreseen that the indium sealing material would extrude so much and cause such a sizeable uncertainty in the results. From the details of the indium measurements presented in appendix $A$, it may be seen that the measurement conditions approximate, but are not identical to, the conditions under which it was used in the experiments. This does not affect the intercomparison of the various results (except that the influence of the atmosphere upon the indium conductance in $1 \mathrm{~F}$ and $2 \mathrm{~A}$ may have been an additional variable), but it does affect the absolute value of the results as compared with theory. If the indium conductance used to correct the data is too small by an order of magnitude, the magnitude of the experimental curves shown in figure 8 could decrease considerably. However, when the sample parameters were varied, but those of the indium were unchanged, as between measurements 1C and 1D, a sizeable change is seen in the conductance before it is corrected for parallel heat flow. This indicates that the indium conductance is not swamping the sample conductance.

A study of the results for sample 1 indicated that the effects observed could be explained by changes in the composition and/or structure of the last few layers of atoms on the solid, rather, than by modifications in the bulk

that were of the order of the phonon wavelength, $\sim 400 \AA$, or larger. It was hypothesized that perhaps lA gave a relatively high conductance compared to $I B$ and $I C$ because the anneal at $480^{\circ} \mathrm{C}$ after the ion bombardment was not at quite a high enough temperature to completely remove the surface-disordering effects resulting from the bombardment, although earlier anneals should have removed the bulk damage. (However, LEED work with anneal temperatures in this range has seemed to produce good surfaces, 65 though much work has been done using higher temperatures. ${ }^{59}$ ) Also, the high conductance observed in measurement ID could be due to the ion bombardment without anneal rather than the deeper damage caused by machining. This ion bombardment was carried out so that foreign atoms could be removed from the surface to the same extent as in other measurements. Furthermore, measurement 1F exhibited a noticeably higher conductance than measurement $1 \mathrm{E}$, yet the conditions were identical, except that the IF surface probably had acquired a cohesive oxide layer ${ }^{64}$ that was very much less than the phonon wavelength. Consequently, measurement $2 \mathrm{C}$ was undertaken to test this hypothesis: The sample would be lightly electropolished (mainly to remove indium adhering from the previous run); given the usual UHV treatment, including a preliminary, outgassing anneal; and given an anneal after the first sequence of ion bombardment to remove any bulk damage that could have been caused by handling the crystal after measurement $2 \mathrm{~B}$. However, the crystal would not be annealed after the second, final ion bombardment. After this procedure had been carried out and helium condensed into the apparatus, an unfortunate accident occurred which prevented the completion of the conductance measurements in the usual manner. The helium level detector in the pumped helium bath became lodged in the coiled tubing through which the helium is condensed to the sample bath, shorted out because of a defect in the insulation, and made a small hole above the liquid level 


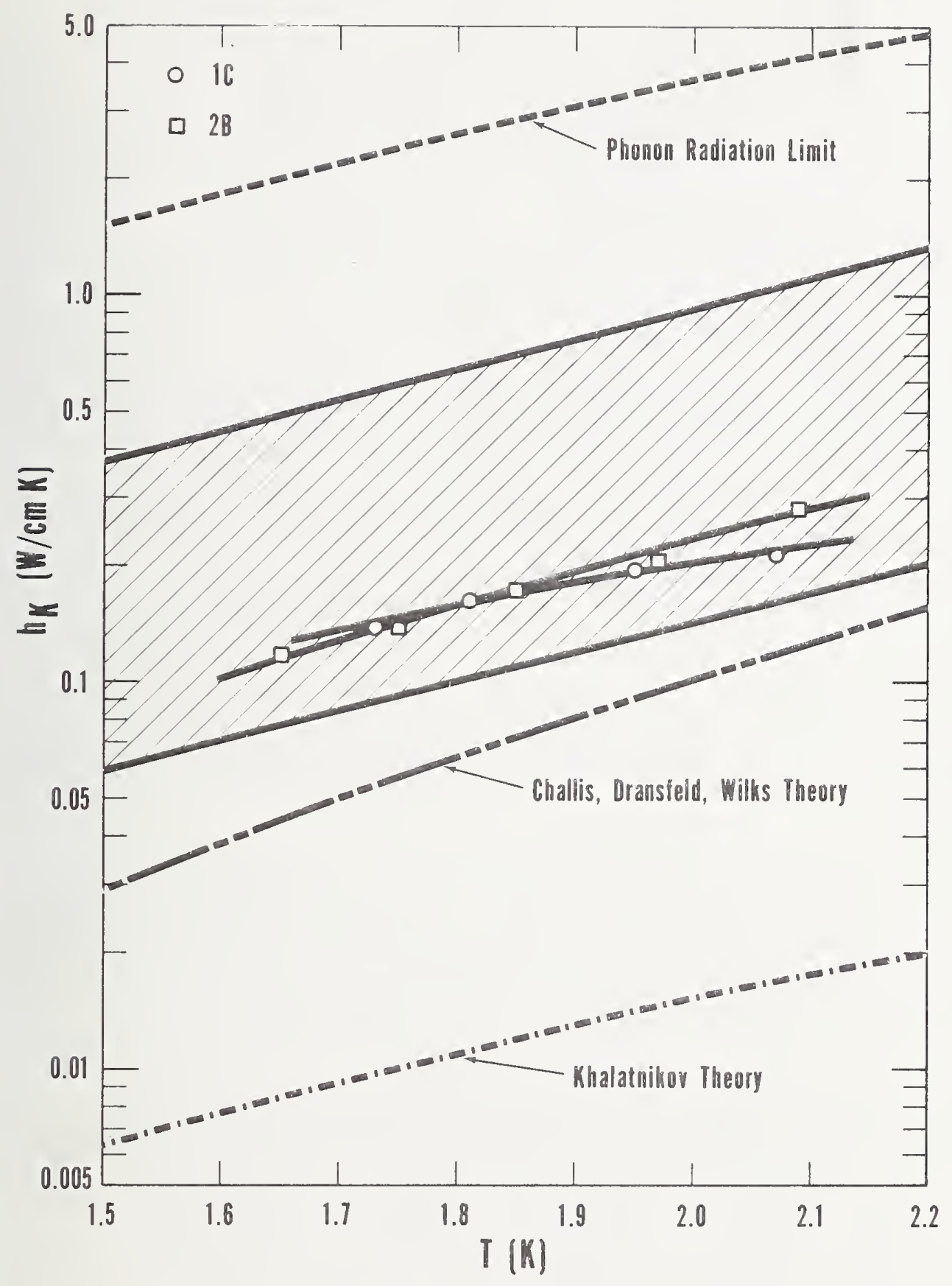

$75 \times 4764$

Figure 9. Comparison of measurements on annealed samples $1 \mathrm{C}$ and 2B. Previous experimental work is represented by the shaded area. (Compare fig. 1) Theoretical curves are explained in the text. 
in the pumped bath. This caused the helium in the sample chamber to be pumped out until, presumably, only a thin film layer of helium II remained. The measurements were attempted anyway and are denoted by the arrows in figure 8. Since the helium film would eventually evaporate as more power was put in, and a very low conductance would then suddenly be observed, the base of the arrow represents a qualitative lower limit, since some finite amount of power had to be used to obtain an observable temperature difference. The measurements do seem to indicate that the conductance had increased markedly; in support of the hypothesis that the last few surface layers are determinate. If this conclusion is correct, it could explain some of the previous disagreements between authors on the effects of surface damage, such as those on nickel, 9,66 since damage to the bulk could be similar, yet differences in the last few atomic layers near the surface could cause significant differences in conductance. If helium atoms exchanging energy with the solid "see" only the outermost layers, the effect of bulk damage could be obscured with oxide layers. Alnaimi and van der sluijs ${ }^{17}$ have observed changes in conductance with the length of time under vacuum which might be due to superficial layers of adsorbed atoms, although the state of the vacuum and surface preparation techniques were not comparable with the present work. Results for samples 1D (damaged) and $1 \mathrm{~B}$ and $1 \mathrm{C}$ (annealed) are in agreement with previous work of Mittag ${ }^{8}$ on polycrystalline samples, also carried out in this laboratory, in a similar temperature range. Although there is apparent agreement between this work and that of Folinsbee and Anderson 12 at 0.03 and $0.3 \mathrm{~K}$ in that coldworking increases the conductance, a closer examination suggests some problems. The temperature dependences observed here, if simply extrapolated to lower temperatures, would cause curves ID and IC to cross, and thus reverse the effect of cold work. Also, since the present results indicate that the condition of the surface layer could be more influential than the state of the bulk, it is difficult to assess agreement with the work at lower temperatures, as deeper layers may be operative at low temperatures, 33 and because surface parameters in the low temperature work were not controlled in a manner similar to this work.

\section{CONCLUSIONS}

In figure 9, the results for the two most ideal surfaces are compared, on a compressed scale, with the original, acoustic mismatch theory of Khalatnikov, eq. (4), the early modifications by challis, et al., 41 and the upper limit for phonon transmission, eq. (3). The shaded portion of the figure shows the range of previous measurements with polycrystalline samples. Though agreement with the acoustic mismatch theory is still lacking by an order of magnitude, the data presented in figures 7 and 8 show that making surface conditions more nearly ideal did lower the conductance by a factor of two or three, towards better agreement with the theory. The large exponent of temperature dependence decreased also to agree somewhat more closely with the theory. In view of the large effect of an oxide layer formed in the atmosphere (measurement lF), and the fact that the monolayer time in storage vacuum of $1 \times 10^{-9}$ torr is less than $1 / 2$ hour, further work under improved vacuum conditions might lower the conductance even more. However, it should be pointed out that the type of oxide layer that would form during exposure to a complete atmosphere is continuous and fairly homogeneous, whereas that formed over a period of time in a vacuum will usually be inhomogeneous ${ }^{67}$ and may have structural differences. 59 It would also be desirable to examine the extent of damage of the material more carefully and to have in situ facilities, such as LEED and Auger instrumentation, for determining the perfection of the surface. It would be expected that some damage would occur near the sealing area. Also, the possible effects of low levels of impurities were not clarified in this study, as the number of samples studied was too low, and time did not permit the study of an extremely pure sample as had been originally planned. Thus, a more definitive test of the theory must await more sophisticated techniques. In this regard, it is interesting to note that the conclusion from the present work that improving sample 
surface conditions lowers the conductance is opposite to our earlier, tentative conclusion based on an examination of the data that was available then. ${ }^{2}$ The difference is presumably that there were no comparisons available earlier for one sample in which only one surface parameter at a time was varied.

There is a strong indication from the present work that the depth of the disorder at the surface need not be comparable to the depth of the phonon wavelength in the solid to have a significant effect, but is more likely to be of the order of a few atomic spacings. Further work in this direction with an even better method of preserving the sample surface, once prepared, would be most desirable. This conclusion is quite important from the practical standpoint of developing good low temperature heat transfer surfaces: If only the outermost layer of the solid need be disordered to improve heat transfer, then there is no need to consider, for instance, extensive coldworking which could seriously impair the thermal conductivity through the bulk, and perhaps not improve the overall situation. From a theoretical standpoint, this conclusion tends to favor a desorption theory of energy transmission, as do the phonon transmission experiments. Surface roughness and adsorbed layers provide mechanisms for differences in energy exchange with incident atoms -- for example, energy exchange increases as the binding energy between adsorbed particles and the lattice decreases, according to calculations at higher temperatures. 27 The conclusion that the last few layers are important in energy exchange may also explain the observation that the temperature dependence is roughly $\mathrm{T}^{2}$ for the cleaner, annealed surfaces: At low temperatures, the specific heat of the surface layer ${ }^{68}$ is proportional to $\mathrm{T}^{2}$, whereas that of the bulk usually goes as $\mathrm{T}^{3}$.

One motivation for this study was the recognition of the difficulty in improving practical heat transfer surfaces without better knowledge of the mechanism of heat transfer at the solid-helium II interface. The wide range of over an order of magnitude for the Kapitza conductance of copper surfaces shown in figure 1 is an indication of the problems encountered when the surface properties are not well-defined. From the results of the present work, it now appears that changing the macroscopic layer of material beneath the surface may have little effect, unless the last few layers of the bulk also happen to be altered by this manipulation. Also, the results indicate that oxide layers could easily overshadow more subtle changes. This effect might well obscure efforts to find out what changes in surfaces are important, if the surfaces are exposed to the atmosphere or an inadequate vacuum before the Kapitza conductance measurement at helium temperatures.

\section{ACKNOWLEDGEMENTS}

The author thanks Dr. V. D. Arp for many useful discussions and for considerable help in the design of the apparatus. Dr. A. Tench kindly carried out the $\mathrm{x}$-ray examinations of the samples. The assistance of G. Wallace was essential. Dr. F. Fickett supplied the RRR determinations. E. Ballinger, W. Decker, and many other staff members were most helpful. 
A sample, $25 \mathrm{~mm}$ in diameter, of the polycrystalline indium used in making o-ring seals was obtained from the Indium Corporation of America. The purity of the indium is quoted as $99.99 \%$. A $25 \mathrm{~mm}$ long portion of this sample was machined off, degreased, and sealed by pressure into the apparatus. The seal leaked slightly below the lambda point, which caused some deterioration of the vacuum and thus an additional heat leak. This resulted in more scatter in the results than usual. Consequently, additional measurements were made in a second, auxiliary apparatus which had previously been used in testing various geometric configurations for the indium seal. The indium sample could be soldered into this apparatus, using a low melting point solder. A schematic sketch of the apparatus is shown in figure 10. Fine lead wires of $0.005 \mathrm{~cm}$ manganin coated with a superconducting lead-tin mixture appeared to isolate the thermometers and heater satisfactorily, although the apparatus was small. After some experimentation with methods of sealing the indium to the apparatus, it was found that soldering with Wood's metal in an oven with a temperature of about $75^{\circ} \mathrm{C}$ would produce a seal that would withstand thermal cycling, and would not leak below the lambda point. Because the thermal contraction of indium is quite large compared to that of most metals, the joint was made to thin stainless which could flex slightly. A further problem was encountered in finding a suitable bonding agent for the heater, since bakeable varnishes which withstand thermal cycling well were unsuitable because of the low melting point of the sample. The clamps on which the thermometers were mounted tended to work loose on the sample, due to extrusion of the soft indium. The poor adhesion of the heater and looseness of clamps sometimes resulted in data which was power dependent. This was eliminated by correcting these problems, and by enclosing the heater with a radiation shield (see fig. 10) so that radiation from the heater which did not thermally equilibrate with the walls of the vacuum enclose could not strike the resistance thermometers.

The results from two measurements each of the first sample, 3 , and a second, longer sample, 4, are shown in figure 11. The results are consistent for each sample (the two measurements with sample 3 were taken over a year apart in different apparatus), but they differ from each other by about 45\%. Because of this consistency, it appears at present that the differences should be ascribed to dissimilar characteristics of the surface of each sample, since there are a number of ways that this may arise during sample preparation. Furthermore, the indium surface encountered in the measurements on copper samples using the indium seals consists chiefly of exposed fresh surface as the indium extruded, so that these results can be expected to give only the order of magnitude of the conductance to be applied as a correction. Several attempts were made to measure the conductance of indium samples whose surfaces were treated in a manner more nearly identical to the o-ring surfaces, but these attempts failed because of the difficulty in making pressure seals with a bulk sample. Etching the surfaces with hydrochloric acid to reduce the oxide layer did not have an effect.

Since previous measurements of indium have not been extensive, it is of interest to compare these measurements with the theory and with previous work. Figure 12 shows that the present measurements are in the range of previous measurements by Gittleman and Bozowski, 69 and Neeper and Dillinger, ${ }^{60}$ though they show a stronger temperature dependence of about $\mathrm{T}^{3.8}$ and $\mathrm{T}^{3.4}$. All experimental results are well above the Khalatnikov acoustic mismatch theory, but not as far above as some metals with higher Debye temperatures have been found to be. Some recent work by Cheeke, et al. 9 on the harder metals has tended to confirm this trend. However, since all measurements have been of polycrystalline samples, measurements on clean, single crystal surfaces would be interesting. Because the samples are polycrystalline and the elastic properties of indium are highly anisotropic, an average value of the transverse velocity of sound was used to obtain the line in figure 12 representing the acoustic mismatch theory. Results for a single crystal would permit a more accurate comparison of theory and experiment, but the handling of such a sample would be a problem. 


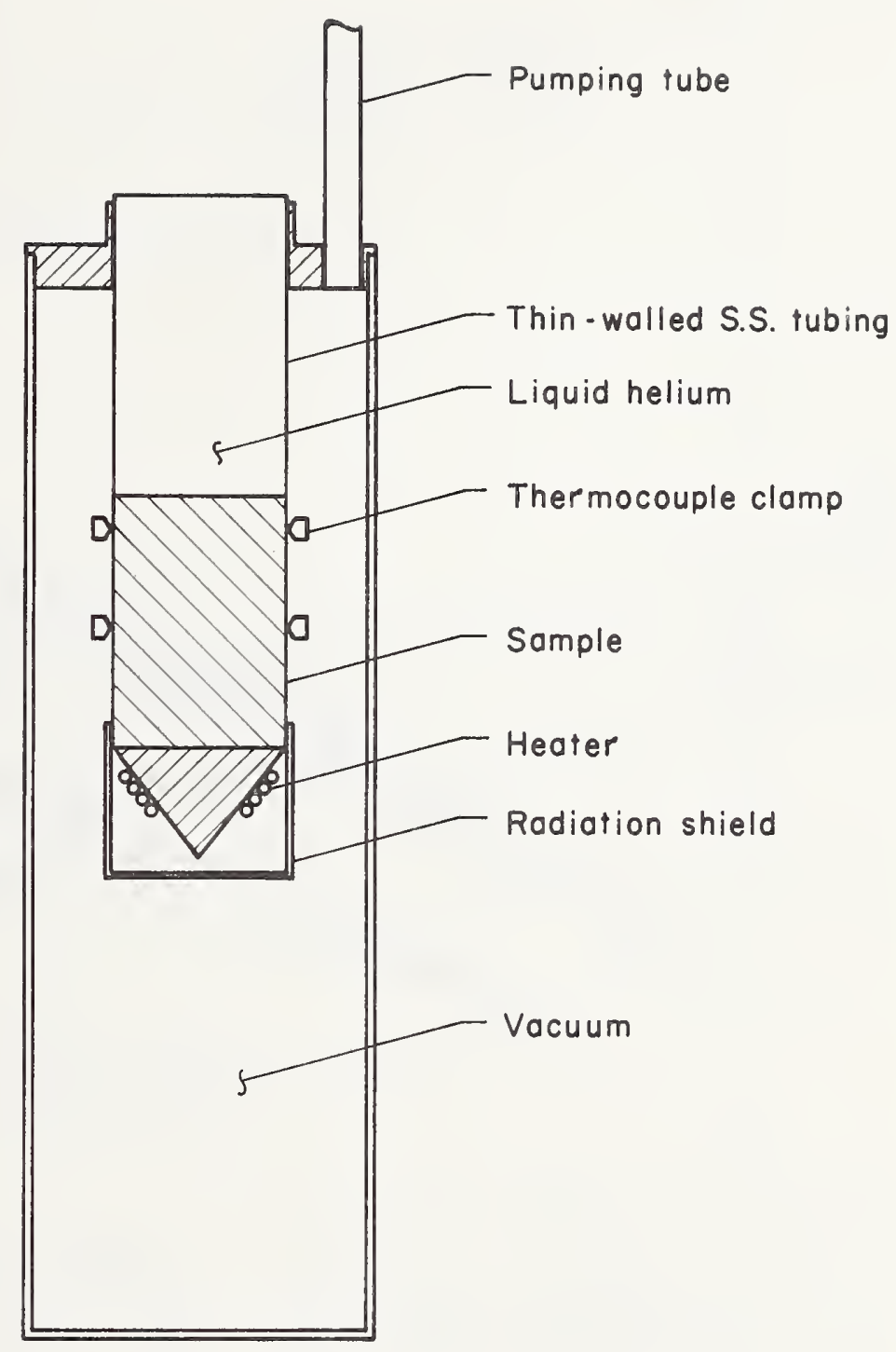

Figure 10. Apparatus for Kapitza conductance measurements of indium. The chamber is placed in a helium bath which is pumped upon to regulate the temperature. 


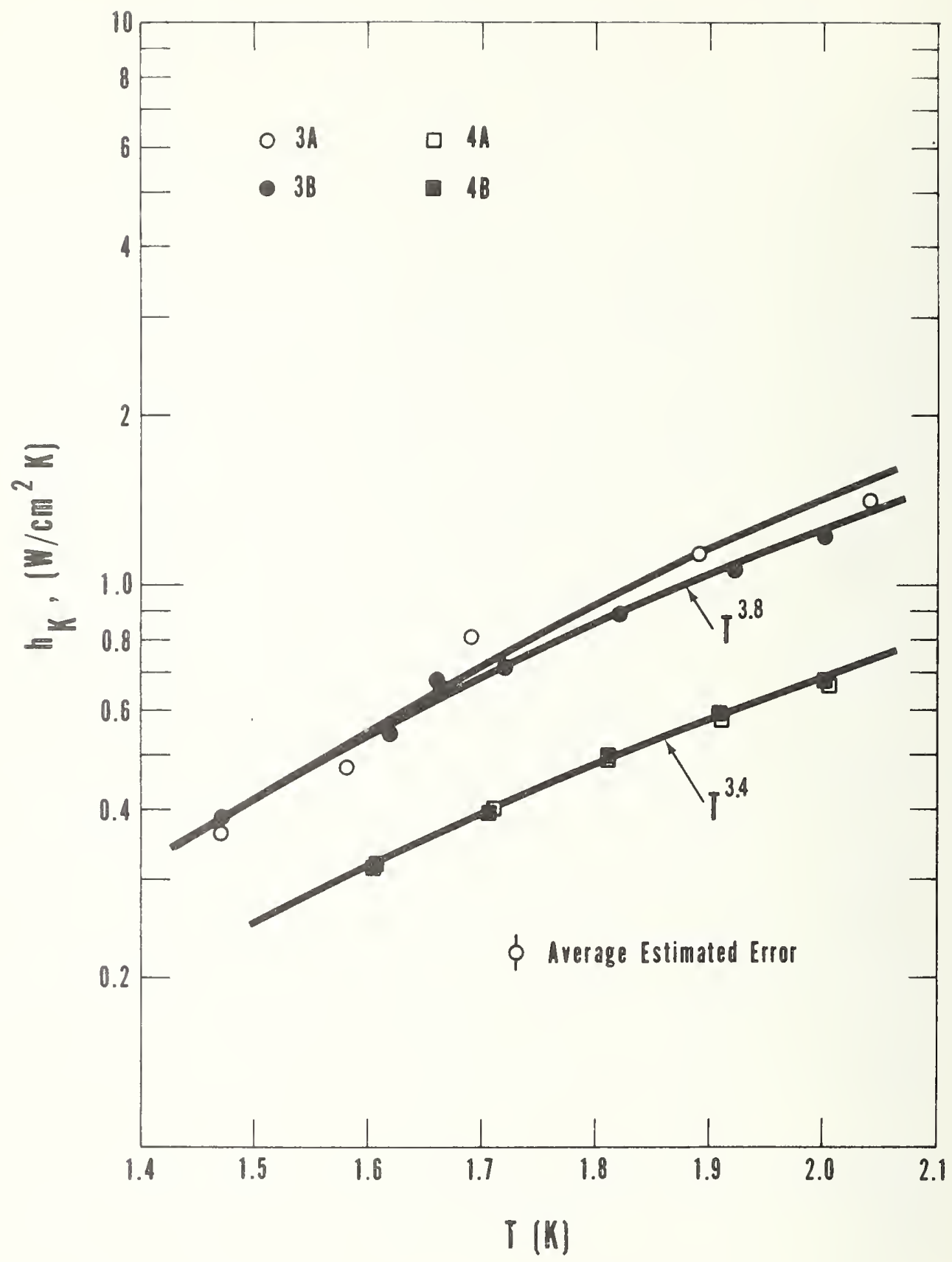

$75 \times 4769$

Figure 11. Kapitza conductance results for two polycrystalline indium samples, 3 and 4. 


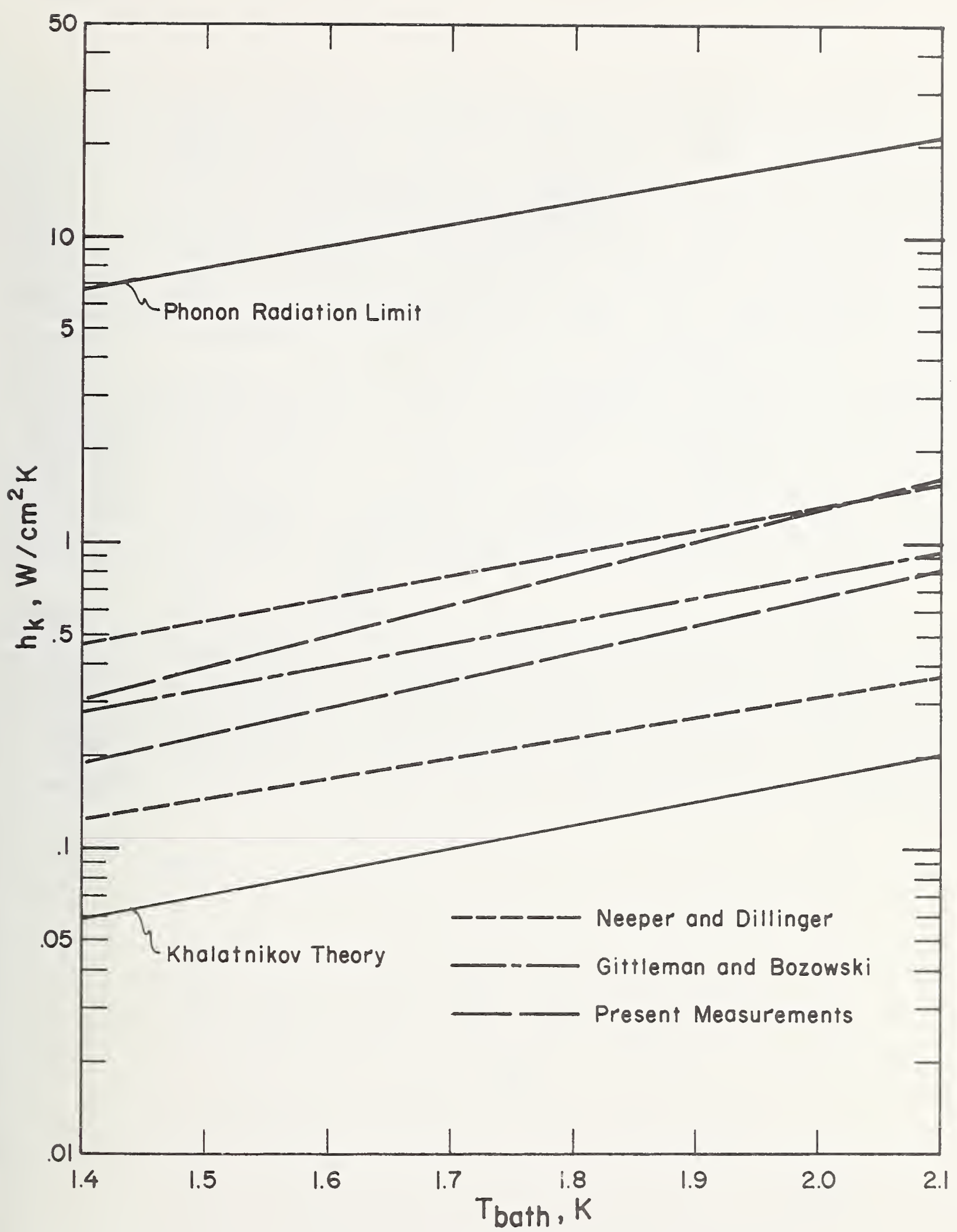

Figure 12. Comparison of Kapitza conducatnce measurements on indium with theory and with previous work. 


\section{APPENDIX B. MEASUREMENT OF THE CONDUCTANCE OF POLYCRYSTALLINE NIOBIUM}

The niobium sample was obtained from the stanford group working on superconducting accelerators (courtesy of Dr. Michael Macashan). The polycrystalIine sample had a preparation similar to that of the superconducting cavities: chemically polished, electropolished, UHV-fired at $1800^{\circ} \mathrm{C}$ at $10^{-9}$ torr. The measurements were made with the sample in the superconducting state. 'In this case, the phonons have a mean free path that is a sizeable fraction of the distance between the thermometers on the sample and between the last thermometer and the interface. Hence, the usual linear extrapolation of temperature to the interface may not be accurate. Therefore, the data from these measurements were analyzed in two ways. Using a linear extrapolation of the temperature measured by the thermometer nearest the interface, a temperature dependence of approximately $\mathrm{T}^{5.4}$ was found for the kapitza conductance. However, if the temperature at the interface is assumed to be most closely approximated by the temperature of the nearest thermometer, because a temperature gradient is not well-defined for distances of the order of a mean free path, the temperature dependence of the present measurements of the Kapitza conductance is somewhat lower, about $T^{4.7}$. Figure 13 shows the results for the case where the temperature is extrapolated to the interface. The error bar represents the uncertainty in the indium correction, as gauged from the present measurements, and the uncertainty of the temperature difference measurement. Figure 13 also shows the same data, but treated differently so that the temperature was not extrapolated to the interface.

A comparison of this data with the phonon radiation limit and the Khalatnikov acoustic mismatch theory is shown in figure 14. Earlier work by Mittag ${ }^{8}$ at this laboratory using a different apparatus is also shown. The curve shown for comparison is the one for which the temperature is extrapolated, as this was the way in which Mittag's data were treated. The sample represented by Mittag's highest curve had a surface preparation which was most similar to the preparation of the sample used in the present work. He also used an indium o-ring seal. It is expected that the disagreement may be due to choosing different values for the indium conductance, since the value Mittag used was not stated. 


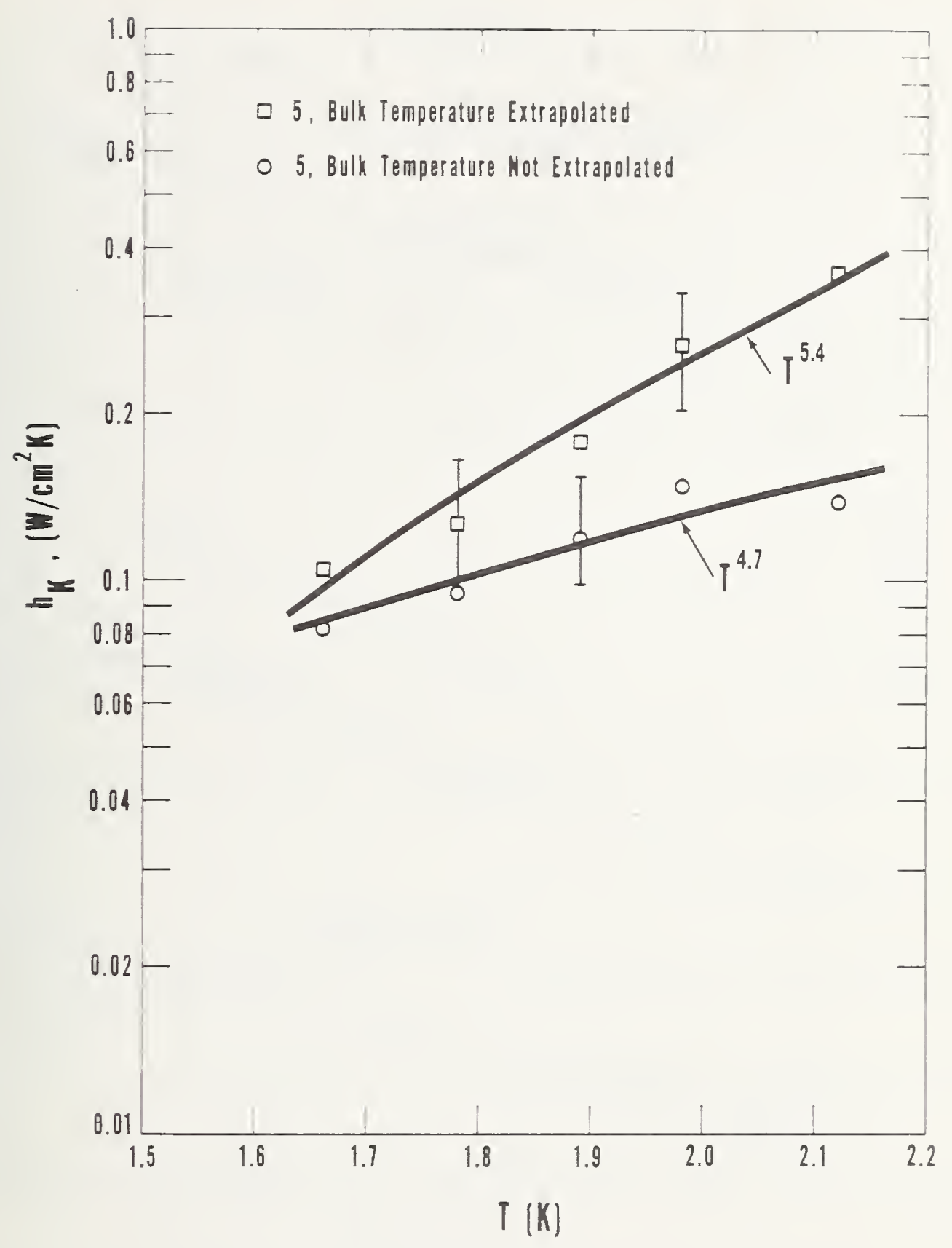

Figure 13. Kapitza conductance of a polycrystalline nlobium sample. Conductance is calculated both by extrapolating the apparent temperature gradient in the sample from the closest thermometer, and by not extrapolating this temperature. 


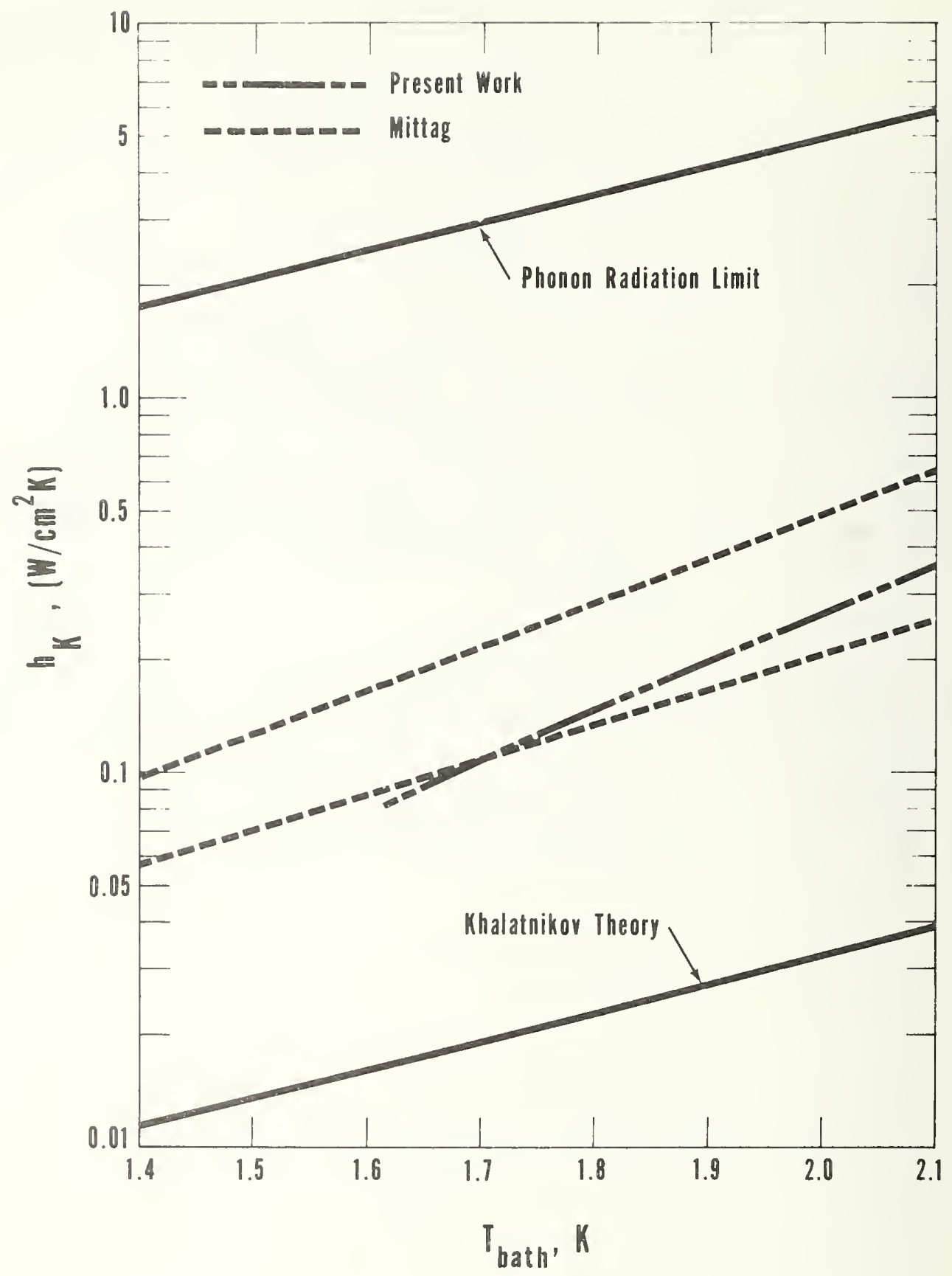

Figure 14. Comparison of the observed Kapitza conductance of niobium with theory and with previous work. The calculation in which the temperature gradient is extrapolated is shown. 


\section{REFERENCES}

1. Kapitza, P. I.., The study of heat transfer in helium II, J. Phys. (Moscow) 4. No. 3, 181-210 (1941).

2. Snyder, N. S., Thermal conductance at the interface of a solid and helium II (Kapitza conductance), Nat. Bur. Stand. (U.S.), Tech. Note 385,90 pages (1969).

3. Wagner, F., Kollarits, F. J., and Yaqub, M., Evidence for the role of conduction electrons in the Kapitza resistance, Phys. Rev. Letters 32, No. 20, 1117-1120 (1974).

4. Frederking, T. H. K., Thermal transport phenomena at liquid helium II temperatures, Chem. Eng. Prog. Symp. Series 64, No. 87, 21-55 (1968).

5. Khalatnikov, I. M., Heat exchange between a solid body and helium II, Zh. Eksp. Teor. Fiz. 22, No. 6, 687-704 (1952).

6. Khalatnikov, I. M., Introduction to the Theory of Superfluidity, Chap. 23 (W. A. Benjamin, Inc., New York 1965).

7. Little, W. A., The transport of heat between dissimilar solids at low temperatures, Can. J. Phys. 37, No. 3, 334-349 (1959).

8. Mittag, K., Kapitza conductance and thermal conductivity of copper, niobium, and aluminum in the range from 1.3 to $2.1 \mathrm{~K}$, Cryogenics 13 , No. 2 , 94-99 (1973).

9. Cheeke, J. D. N., Hebral, B., and Richard, J., Kapitza resistance between the transition metals $\mathrm{Fe}, \mathrm{Co}$, and $\mathrm{Ni}$ and superfluid helium, J. Low Temp. Phys. 12, Nos. 3/4, 359-373 (1973).

10. Pollack, G. I., Kapitza resistance, Rev. Mod. Phys. 41, No. 1, 48-81 (1969).

11. Snyder, N. S., Heat transport to helium II: Kapitza conductance, Cryogenics 10 , No. 2 , 89-95 (1970).

12. Folinsbee, J. T. and Anderson, A. C., The Kapitza resistance to a variety of metallic surfaces below $0.3 \mathrm{~K}$, J. Low Temp. Phys. 17, Nos.5/6, 409424 (1974).

13. Challis, I. J. and Cheeke, J. D. N., The Kapitza conductance in lead, Prog. in Refrig. Sci. and Tech., Vol. I, 227-230 (Pergamon Press, London, 1965 ).

14. Johnson, R. C. and Iittle, W. A., Experiments on the Kapitza resistance, Phys. Rev. 130, No. 2, 596-604 (1963).

15. Johnson, R. C., Kapitza resistance of a crystal cleaved while immersed in liquid helium, Bull. Am. Phys. Soc. 9, No. 7, 713 (1964).

16. Anderson, A. C. and Johnson, W. I., The Kapitza resistance between copper and ${ }^{3}$ He, J. Low Temp. Phys. 7, Nos. $1 / 2,1-21$ (1972).

17. Alnaimi, A. E. and van der Sluijs, J. C. A., On the experimental reproducibility in measurements of the Kapitza conductance of copper between 1 and $2 \mathrm{~K}$, Cryogenics 13, No. 12, 722-725 (1973).

18. Peterson, R. E. and Anderson, A. C., The transport of heat between solids at low temperatures, Solid state Commun. 10, No. 9, 891 (1972). 
19. Cheeke, J. D. N., Hebral, B., and Martinon, C., Transfer de chaleur entre deux solides en dessous de $100 \mathrm{~K}$ (Heat transfer between two solids below 100 K), J. Physique $34,257-272$ (1973).

20. Mezhov-Deglin, L. P., Measurement of the thermal conductivity of crystalline $\mathrm{He}^{4}$, Soviet Phys.-JETP 22, No. 1, 47-56, Trans. from Zh. Eksperim. i. Teor. Fiz. 49, 66-79 (1965).

21. Cheeke, J. D. N. and Hebral, B., Comments on the Kapitza conductance of copper to liquid and solid ${ }^{4} \mathrm{He}$, Phys. Letters 40A, No. 4, 301-302 (1972).

22. Folinsbee, J. T. and Anderson, A. C., Anomalous Kapitza resistance to solid helium, Phys. Rev. Letters 31, No. 27, 1580-1581 (1973).

23. Guo, C. J. and Maris, H. J., Experimental study of the reflection of phonons at an interface between dielectric crystals and liquid helium, gaseous helium, and solid neon, Phys. Rev. Al0, No. 3, 960-966 (1974).

24. Long, A. R., Sherlock, R. A., and Wyatt, A. F. G., Phonon reflection at a cleaved sodium fluoride-helium film interface, J. Low Temp. Phys. 15, Nos. 5/6, 523-536 (1974).

25. Kinder, $\mathrm{H}$. and Dietsche, W., Strong phonon conversion at the heliumsolid interface, Phys. Rev. Letters 33, No. 10, 578-581 (1974).

26. Haug, H., Transmission probabilities of high-frequency phonons through a solid-He II interface, Phys. Letters 45A, No. 2, 170-172 (1973).

27. Lorenzen, J. and Raff, L. M., Theoretical investigations of gas-solid interaction phenomena. III. Adsorbed particles and lattice impurities. J. Chem. Phys. 52, No. 3, 1133-43 (Feb. 1, 1970).

28. Sherlock, R. A., Wyatt, A. F. G., Mills, N. G., and Lockerbie, N. A., Angular distribution of phonons radiated from a heated solid into liquid ${ }^{4} \mathrm{He}$, Phys. Rev. Letters 29, No. 19, 1299-1301 (1972).

29. Narnhofer, H., Thirring, W., and Sexl, R., On the theory of interfacial conductivity, in Thermal Conductivity - Proceedings of the Eighth Conf., 161 (Plenum Press, New York, 1969).

30. Challis, L. J. and Cheeke, J. D. N., Some comments on the theory of the Kapitza conductance between metals and liquid helium II, Proc. Roy. Soc. A 304, No. 1479,479-486 (1968).

31. Andreev, A. F., The effect of conducting electrons on the Kapitza temperature jump, Soviet Phys.-JETP 16, No. 4, 1084-1088, Trans. from Zh. Eksp. Teor. Fiz. 43, No. 4, 1535- $\overline{15} 42$ (1962).

32. Khalatnikov, I. M. and Adamenko, I. N., Theory of the Kapitza temperature discontinuity at a solid body-liquid helium boundary, soviet Phys.JETP 36, No. 3, 391-394, Trans. from Zh. Eksp. Teor. Fiz. 63, 746-752 $(1 9 7 2 \longdiv { \text { . } }$

33. Peterson, R. E. and Anderson, A. C., The Kapitza thermal boundary resistance, J. Low Temp. Phys. 11, Nos. 5/6, 639-665 (1973).

34. Haug, H. and Weiss, K., A modified theory of the Kapitza resistance, Phys. Letters 40A, No. 1, 19-20 (1972).

35. Vuorio, M., On the existence of the critical cone in Kapitza resistance experiments, J. Low Temp. Phys. 10, Nos. 5/6, 781-785 (1973). 
36. Challis, L. J., Kapitza resistance and acoustic transmission across boundaries at high frequencies, J. Phys. C: Sol. State Phys. 7, No. 3, $481-495$ (1974).

37. Sluckin, T. J., Toombs, G. A., and Sheard, F. W., The effect of phonon life times on the Kapitza resistance, Solid State Commun. 14, No. 2, 203-207 (1974).

38. Cheeke, J. D. N., Some experimental investigations of the Kapitza resistance of lead, Cryogenics 10, No. 12, 463-472 (1970).

39. Little, W. A., Unimportance of surface roughness upon the Kapitza resistance, Phys. Rev. 123, No. 6, 1909-1911 (1961).

40. Adamenko, I. N. and Fuks, I. M., Roughness and thermal resistance of the boundary between a solid and liquid helium, Soviet Phys.-JETP 32, No. 6 , 1123-1129, Trans. from Zh. Eksp. Teor. Fiz. 59, 2071-2082 (1970).

41. Challis, L. J., Dransfeld, K., and Wilks, J., Heat transfer between solids and liquid helium II, Proc. Roy. Soc. A260, No. 1300, 31-46 (1961).

42. Toombs, G. A. and Challis, L. J., Kapitza conductance due to helium atoms interacting with a surface, J. Phys. C: Solid St. Phys. $\underline{4}$, No. 10, 1085-1909 (1971).

43. Vuorio, M., The effect of low energy vibrational modes on the Kapitza resistance, J. Phys. C: Solid St. Phys. ㅁ, No. 11, 1216-1221 (1972).

44. Long, A. R., Energy transfer from solids to helium vapor by the desorption of surface-bound atoms, J. Low Temp. Phys. 17, Nos. 1/2, 7-23 (1974).

45. Weiss, K., in Proc. of the German Physical Society 1974 Spring Conf., Freudenstadt (to be published).

46. Opsal, J. I. and Pollack, G. L., Improved calculations of Kapitza resistance: Combined effects of phonon attenuation and impedance matching on Kapitza resistance, Phys. Rev. A 9, No. 5, 2227-2235 (1974).

47. Radebaugh, R., Siegwarth, J. D., and Holste, J. C., Private communication.

48. Fairbank, H. A. and Wilks, J., Heat Transfer in liquid helium below $1^{\circ} \mathrm{K}$, Proc. Roy. Soc. A231, No. 1187, 545-555 (1955).

49. Saslow, W. M., Shear-wave dissipation and the Kapitza conductance, J. Low Temp. Phys. 11, Nos. 3/4, 255-275 (1973).

50. Reid, R. J., LEED surface Debye temperature of Cu(100), Phys. Status Solidi A 2 , No. 2, 109-111 (1970).

51. Jona, F., Low energy electron diffraction study of surfaces of antimony and bismuth, Surface Sci. 8, Nos. 1/2, 57-76 (1969).

52. McCracken, G. M., The performance of a high speed getter pump using a cooled titanium film, Vacuum 15, No. 9, 433-436 (1965).

53. Young, F. W., Jr. and Wilson, T. R., Acid cutting and acid polishing of copper crystals, Rev. Sci. Instr. 32, No. 5, 559-562 (1961).

54. Gniewek, J. J., Clark, A. F., and Moulder, J. C., Spark planing damage in copper, Nat. Bur. Stand. (U.S.), Tech. Note 321,6 pages (1965). 
55. Powers, R. W., The electropolishing of specimens, Electrochemical Technology 2 , No. 9-10, 274-281 (1964).

56. Stuart, R. V., sputtering yields at medium and low energies, Trans. of the Eighth National Vacuum Symp., ed. L. E. Preuss, 1, 252-258 (Pergamon Press, New York, 1961).

57. Steiger, R. F., Morabito, Jr., J. M., Somorjai, G. A., and Muller, R. H., A study of the optical properties and of the physical adsorption of gases on silver single crystal surfaces by low energy electron diffraction and ellipsometry, Surface Sci. 14, No. 2, 279-304 (1969); and comments, 20, 187-189; reply to, 20, 190-191 (1970).

58. Rhodin, Jr., T. N., Low temperature oxidation of copper. I. Physical mechanism, J. Amer. Chem. Soc. 72, No. 11, 5102-5106 (1950).

59. Lee, R. N. and Farnsworth, H. E., LEED studies of adsorption on clean (100) copper surfaces, Surface Sci. 3, No. 5, 461-479 (1965).

60. Neeper, D. A. and Dillinger, J. R., Thermal resistance at indium-sapphire boundaries between 1.1 and 2.1 K, Phys. Rev. 135, No. 4A, 1028-1033, (1964).

61. Avrami, M., and Little, J. B., Diffusion of heat through a rectangular bar and the cooling and insulating effect of fins. I. The steady state, J. Appl. Phys. 13, 255-264 (1942).

62. Clark, A. F., Deason, V. A., Hust, J. G., and Powell, R. L., The eddy current decay method for resistivity characterization of high purity metals, Nat. Bur. Stand. (U.S.) Spec. Publ. 260-39, 43 pages (1972).

63. Cheeke, J. D. N., Hebral, B., Richard, J., and Turkington, R. R., Anomalous behavior of the Kapitza resistance between solids and liquid helium II, Phys. Rev. Letters 32, No. 12, 658-661 (1974).

64. Evans, U. R., The Corrosion and Oxidation of Metals: Scientific Principles and Practical Applications, Chap. II, (Edward Arnold, Ltd., London, 1960).

65. Andersson, S., Low energy electron diffraction intensities from the clean copper (001) surface, Surface Sci. 18, No. 2, 325-340 (1969).

66. Wey-Yen, K., An investigation of the temperature discontinuity at the boundary between a solid and superfluid helium, Soviet Phys.-JETP 15, No. 4, 635-645, Transl. from Zh. Eksp. Teor. Fiz. 42, No. 4, $921-935$ (1962).

67. Lawless, K. R., The oxidation of metals, Rep. Prog. Phys (GB) 37, No. 2, $231-316(1974)$.

68. Wallis, R. F., Lattice dynamics of crystal surfaces, Prog. in Surface Sci. 4, Part 3, 233-367 (Pergamon Press, Oxford, 1973).

69. Gittleman, J. I. and Bozowski, S., Some aspects of the Kapitza resistance, Phys. Rev. 128, No. 2, 646-367 (Pergamon Press, Oxford, 1973). 
Director

Advanced Research Projects Agency

I copy

Attn: Technical Library

1400 Wilson Boulevard

Arlington, Virginia 22209

Office of Naval Research

Electronics Program Office (Code 427)

1 copy

800 North Quincy Street

Arlington, Virginia 22217

Office of Naval Research

Code 105

800 North Quincy Street

Arlington, Virginia 22217

Naval Research Iaboratory

6 copies

Department of the Navy

Attn: Code 2627

Washington, D. C. 20375

Office of the Director of Defense Research and Engineering

1 copy

Information Office Iibrary Branch

The Pentagon

Washington, D. C. 20301

U. S. Army Research Office

6 copies

Box CY, Duke Station

Durham, North Carolina 27706

Defense Documentation Center

12 copies

Cameron Station

Blexanciria, virginia 22314

Director, National Bureau of Standards

1 copy

ìtn: Technical Library

iashington, D. C. 20234

Commanding officer

OEice of Naval Research Branch Office

I copy

536 South Clark Street

Cnicago, Illinois 60605

San Francisco Frea Office

1 copy

Cffice of Naval Research

50 Eell Street

San Francisco, California 94102

Fir Eorce Office of Scientific Research

I copy

Department of the Air Force

iashington, D. C. 20333

Commanding officer

OEflce of Naval Research Branch Office

1 copy

1030 Eas= Green Street

Fasadena, California 91101

Commanding officer

1 copy

Office of Naval Research Branch Office

495 Summer Street

Boston, Massachusetts

02210 
Fort Belvoir, Virginia 22060

Attn: Technical Documents Center

ODDR\&E Advisory Group on Electron Devices

1 copy

201 Varick Street

New York, New York 10014

New York Area Office

1 copy

Office of Naval Research

207 West 24 th Street

New York, New York 10011

Air Force Weapons Laboratory

1 copy

Technical Library

Kirtland Air Force Base

Albuquerque, New Mexico

87117

Air Force Avionics Laboratory

1 copy

Air Force Systems Command

Technical Library

Wright-Patterson Air Force Base

Dayton, Ohio 45433

Air Force Cambridge Research Laboratory

1 copy

L. G. Hanscom Field

Technical Library

Cambridge, Massachusetts 02138

Harry Diamond Laboratories

1 copy

Technical Library

Connecticut Avenue at Van Ness, N.W.

Washington, D. C. 20438

Naval Air Development Center

Attn: Technical Library

Johnsville

Warminster, Pennsylvania 18974

Naval Weapons Center

Technical Library (Code 753)

1 copy

China Lake, California 93555

Naval Training Device Center

Technical Library

Orlando, Florida 22813

Naval Research Laboratory

Underwater Sound Reference Division

1 copy

r'echnical Library

P. O. Box 8337

Orlando, Florida 32806

Navy Underwater Sound Laboratory

1 copy

Technical Library

Fort Trumbull

New London, Connecticut 06320

Commandant, Marine Corps

Scientific Advisor (Code AX)

1 copy

Washington, D. C. 20380

1 copy 
Philadelphia Division

Technical Library

Philadelphia, Pennsylvania 19112

Naval Postgraduate School

1 copy

Technical Library (Code 0212)

Monterey, California 93940

Naval Missile Center

Technical Library (Code 5632.2)

1 copy

Point Mugu, California 93010

Naval Ordnance Station

Technical Library

1 copy

Louisville, Kentucky 40214

Naval Oceanographic Office

Technical Library (Code 1640)

1 copy

Suitland, Maryland 20390

Naval Explosive Ordnance Disposal Facility

1 copy

Technical Library

Indian Head, Maryland 20640

Naval Electronics Laboratory Center

Technical Library

1 copy

San Diego, California 92152

Naval Undersea Warfare Center

Technical Library

1 copy

3202 East Foothill Boulevard

Pasadena, California 91107

Naval Weapons Laboratory

1 copy

Technical Library

Dahlgren, Virginia 22448

Naval Ship Research and Development Center

Central Library (Code L42 and L43)

Washington, D. C. 20007

Naval Ordnance Laboratory White Oak

Technical Library

Silver Spring, Maryland 20910

Naval Avionics Facility

Technical Library

Indianapolis, Indiana 46218 
NBS-114A (REV. 7-73)

\begin{tabular}{|c|c|c|c|}
\hline $\begin{array}{l}\text { U.S. DEPT. OF COMM. } \\
\text { BIBLIOGRAPHIC DATA } \\
\text { SHEET }\end{array}$ & $\begin{array}{l}\text { 1. PUBLICATION OR REPORT NO. } \\
75-812\end{array}$ & $\begin{array}{l}\text { 2. Gov't Accession } \\
\text { No. }\end{array}$ & 3. Recipient's Accession No. \\
\hline \multirow{2}{*}{\multicolumn{3}{|c|}{$\begin{array}{l}\text { 4. TITLE AND SUBTITLE } \\
\text { THE KAPITZA CONDUCTANCE OF THE (100) SURFACE OF COPPER AND } \\
\text { POLYCRYSTALLINE INDIUM AND NIOBIUM }\end{array}$}} & $\begin{array}{l}\text { 5. Publication Date } \\
\text { June } 1975\end{array}$ \\
\hline & & & 6. Performing Organization Code \\
\hline \multicolumn{3}{|c|}{$\begin{array}{l}\text { 7. AUTHOR(S) } \\
\text { N. S. Snyder }\end{array}$} & $\begin{array}{l}\text { 8. Performing Organ. Report No. } \\
\text { NBSIR 75-812 }\end{array}$ \\
\hline \multirow{2}{*}{\multicolumn{3}{|c|}{$\begin{array}{l}\text { 9. PERFORMING ORGANIZATION NAME AND ADDRESS } \\
\qquad \begin{array}{l}\text { NATIONAL BUREAU OF STANDARDS } \\
\text { DEPARTMENT OF COMMERCE } \\
\text { WASHINGTON, D.C. } 20234\end{array}\end{array}$}} & $\begin{array}{l}\text { 10. Project/Task/Work Unit No. } \\
2750187\end{array}$ \\
\hline & & & $\begin{array}{l}\text { 11. Contract/Grant No. } \\
\text { NAonr }-22-74\end{array}$ \\
\hline \multirow{2}{*}{\multicolumn{3}{|c|}{$\begin{array}{l}\text { 12. Sponsoring Organization Name and Complete Address (Street, City, State, ZIP) } \\
\text { Office of Naval Research } \\
\text { Arlington, VA } 22217\end{array}$}} & $\begin{array}{l}\text { 13. Type of Report \& Period } \\
\text { Covered }\end{array}$ \\
\hline & & & 14. Sponsoring Agency Code \\
\hline
\end{tabular}

15. SUPPLEMENTARY NOTES

16. ABSTRACT (A 200-word or less factual summary of most significant in formation. If document includes a significant bibliography or literature survey, mention it here.)

Measurements of the Kapitza conductance to liquid helium II across the (100) surface of single crystals of copper are presented. The temperature range of these measurements was 1.6 to $2.1 \mathrm{~K}$.

The sample surfaces were subjected to several different treatments. Some surfaces were cleaned by low energy argon ion bombardment, annealed in an ultrahigh vacuum system, and preserved under vacuum until purified liquid helium was admitted. Other surfaces were intentionally damaged by machining and/or exposure to the atmosphere. The conductance after these latter treatments was found to be about a factor of three higher than that of the more ideally cleaned and annealed surfaces, and a significant increase in the temperature dependence of the conductance was also observed. Indications are that these effects on the conductance are produced primarily by the last few atomic layers of the solid, so extensive cold-working, which impairs the thermal conductivity of the bulk, need not be undertaken to improve surface heat transfer. This finding has important implications for the design of practical heat transfer surfaces. Good reproducibility was found for the conductances of similarly treated sufaces and good correlation with studies of damage carried out by $x-r a y$ diffraction. The relationship of these results to the numerous current theories of the Kapitza conductance is discussed. Conductance measurements of polycrystalline niobium and indium are presented in the appendices.

17. KEY WORDS (six to twelve entries; alphabetical order; capitalize only the first letter of the first key word unless a proper name; separated by semicolons) Heat transfer to helium II; interfacial thermal resistance; Kapitza conductance; Kapitza conductance of copper; Kapitza conductance of indium; Kapitza conductance of niobium; (100) surface of copper; x-ray diffraction examination of single crystal lattice damage.

18. AVAILABILITY $\mathrm{X}$ Unlimited

For Official Distribution. Do Not Release to NTIS

Order From Sup. of Doc., U.S. Government Printing Office Washington, D.C. 20402, SD Cat. No. C13

$\mathrm{X}$ Order From National Technical Information Service (NTIS) Springfield, Virginia 22151

\begin{tabular}{|c|c|}
\hline $\begin{array}{l}\text { 19. SECURITY CLASS } \\
\text { (THIS REPURT) }\end{array}$ & $\begin{array}{l}\text { 21. NO. OF PAGES } \\
50\end{array}$ \\
\hline $\begin{array}{l}\text { 20. SECURITY CLASS } \\
\text { (THIS PAGE) } \\
\text { UNCLASSIFIED }\end{array}$ & $\begin{aligned} \text { 22. } & \text { Price } \\
& \$ 3.75\end{aligned}$ \\
\hline
\end{tabular}

